Apidologie, 1974, 5 (2), 149-175.

\title{
DER EINFLUSS SUBLETALER DOSEN VON PARATHION (E 605) AUF DIE ENTFERNUNGSWEISUNG BEI DER HONIGBIENE
}

\author{
L'influence de doses sublétales de parathion ( $\mathrm{E} 605$ ) \\ sur l'indication de la distance chez l'abeille
}

\author{
Burkhard SCHRICKER ${ }^{1}$ \\ Institut für Allgemeine Zoologie \\ und Experimentelle Morphologie der \\ Freien Universität Berlin
}

\begin{abstract}
SUMMARY
THE EFFECT OF SUblethal DOSES OF PARATHion (E 605)

ON THE INDICATION OF DISTANCE IN HONEYBEES
\end{abstract}

The sublethal dose of Parathion (E 605) applied orally in these experiments was less than 0.03 Gamma. After a treatment with Parathion foraging bees trained to an artificial feeding site indicated the distance to the hive too short by means of an increased waggingdance-rhythm. There is no difference, whether those dances are performed on a comb in vertical or in horizontal position. Recruits interpret the misinformation given by the dances of poisoned bees in a c correct " manner, and so they are misled. Foragers trained to an artificial feeding site were poisoned there and immediately transferred to an unknown area : there they tried to locate the feeding site in a shorter distance. In phototactic runs towards an artificial light source the speed of poisoned bees was increased by about 10 per cent. The effect of a sublethal dose of Parathion on the dance-rhythm diminishes about 2.5 hours after treatment, and it is no further detectable after 5.5 hours. A probable mode of action of Parathion is discussed as well as a probable correlation between dance-rhythm and measuring the distance.

1. Anschrift : Priv. Doz. Dr. B. Schricker, Inst. für Allgem. Zoologie und Exper. Morphol., 1 Berlin-33 Königin Luise Straße 1-3. 


\section{ZUSAMMENFASSUNG}

Die in diesen Versuchen peroral verabfolgte subletale Giftmenge von Parathion beträgt < 0,03 Gamma. Zu einer künstlichen Futterquelle dressierte Sammlerinnen geben deren Entfernung zum Stock nach einer Vergiftung zu kurz an, ausgedrückt in einem erhöhten Tanztempo. Dabei besteht kein Unterschied, ob die Tänze auf vertikaler oder auf horizontaler Wabe ausgeführt werden. Neulinge erkennen die Tänze vergifteter Sammlerinnen nicht als verändert und werden entsprechend fehlgeleitet. Sammlerinnen, die auf eine künstliche Futterquelle gut eingeflogen sind, werden am Futterplatz vergiftet und unmittelbar darauf in ein fremdes Gelände versetzt. Dort suchen sie die Futterquelle ebenfalls in einer kürzeren Entfernung. Bei phototaktischen Läufen zu einer künstlichen Lichtquelle ist die Laufgeschwindigkeit vergifteter Bienen gegenüber der im unvergifteten Zustand um etwa $10 \%$ erhöht. Die Wirkung einer Subletaldosis Parathion auf das Tanztempo läßt etwa 2 1/2 Stunchen nach der Vergiftung nach und ist nach $51 / 2$ Stunden nicht mehr festzustellen. Der mögliche Wirkungsmechanismus des Parathion und der Zusammenhang zwischen Tanztempo und Entfernungsweisung werden diskutiert.

\section{I. - EINLEITUNG}

Diese Untersuchungen gehen von der Frage aus, inwieweit subletale Dosen von Insektiziden in den Lebensablauf eines tierischen Organismus einwirken - Giftmengen in Bruchteilen eines millionstel Grammes, wie sie als Rückstände überall in der Natur anzutreffen sind (Carson 1962, Newsom 1967). Zu diesem Problem sind bisher nur wenige verhaltensphysiologische Untersuchungen an Arthropoden gemacht worden, hauptsächlich im weiteren Bereich der neurotropen Substanzen. So stellten WITT u. REED (1965) bei Webespinnen einen ungeordneten Netzbau fest (Zusammenfassung : Witt, Reed u. Peakall 1968). Stephen u. Young (1970) fanden bei Männchen der Hausgrille (Acheta domesticus) veränderte Lautäußerungen im Werbegesang. In beiden Fällen wirken subletale Dosen auf sehr komplexe Verhaltensweisen ein, d. h. hierbei sind mehrere Sinnesorgansysteme beteiligt und greifen ineinander.

Aus eben diesen Gründen schien uns der Schwänzeltanz der Honigbiene für derartige Untersuchungen wegen seiner Komplexeität gut geeignet zu sein. Mit ihm werden sowohl die Richtung als auch die Entfernung einer Futterquelle angezeigt, wobei neben optischen und mechanischen Orientierungskomponenten auch die zum Sammelflug aufgewendete Energie gewertet wird. In früheren Untersuchungen (Schricker u. StePhen 1970, Stephen u. SchriCKER 1970) konnten wir feststellen, daß Sammelbienen nach einer peroralen oder topikalen Vergiftung bei ihrer Richtungsweisung auf der Wabe in Vertikalposition typisch veränderte Tanzwinkel angeben.

Im Folgenden soll untersucht werden, ob und inwieweit auch Veränderungen in der Entfernungsweisung nach einer subletalen Vergiftung auftreten.

\section{II. - MATERIAL UND METHODE}

Untersucht wurden Sammelbienen von Apis mellifica carnica. Die Giftdosis wurde stets peroral (in 2-molarer Zuckerlösung) verabfolgt.

In jedem Versuch wird eine Gruppe individuell markierter Bienen aus einem Volk zu einer künstlichen Futterquelle dressiert, die je nach Versuchsreihe 185 Meter, 530 Meter und 865 Meter vom Stock entfernt liegt. Die Sammlerinnen dürfen sich jeweils mindestens 3 Tage lang auf die Futterquelle einfliegen. Die Fütterungszeit beträgt dazu täglich 1 Stunde. Beim kritischen Versuch wird im Stock das Tanztempo der heimgekehrten Sammlerinnen gemessen, das ist die Zeit, die für jeweils 10 Tanzumläufe auf der Wabe in Vertikalposition bzw. in Horizontalposition benötigt wird. Am Futterplatz eintreffende Neulinge werden vom Beobachter abgefangen und in Alkohol abgetötet. Nachdem das Tanztempo eine Zeitlang bestimmt worden ist, wird kurz vor dem Zeitpunkt der Vergiftung die Hälfte der Dressurschar am Futter- 
platz abgefangen und vorübergehend gekäfigt. Der genaue Zeitpunkt der Vergiftung wird von dem Beobachter am Futterplatz festgelegt und bleibt - ebenso wie die Entscheidung über die Auswahl der zu vergiftenden Bienen — dem Beobachter am Stock bis nach dem Versuchsende unbekannt. Der nicht gekäfigten Hälfte der Bienenschar wird in einem anderen Futtergefä $\beta$ eine einmalige Gabe vergifteten 2 - molaren Zuckerwassers (Giftkonzentration $=$ 5 ppm. Bestimmung der subletalen Dosis : Siehe unten) geboten. Nach der Vergiftung, die gewöhnlich innerhalb von zwei bis drei Minuten abgeschlossen ist, wird das Futtergefä $\beta$ gegen das ursprüngliche ausgetauscht. Die unvergifteten Kontrollbienen werden unverzüglich wieder freigelassen, soda $\beta$ beide Gruppen das Sammeln wiederaufnehmen können. Im Stock wird weiterhin das Tanztempo der vergifteten Bienen und der Kontrollbienen gemessen.

\section{Bestimmung der Subletaldosis von Parathion (E 605):}

Bei der Bestimmung der Subletaldosis gingen wir von den Ergebnissen aus, die SchIck (1953) bei Sammelbienen gewonnnen hat. Er hatte Parathion in 2-molarer Zuckerlösung in einer Wirkstoffkonzentration von $40 \mathrm{ppm}$ als Emulsion geboten, da die Löslichkeit von Parathion in Wasser bei Zimmertemperatur etwa 20 Gamma pro Milliliter $=20$ ppm (PERKow 1956) beträgt. Schick zeigte in seinen Untersuchungen zur Wirkung verschiedener Gifte auf Bienentänze, daß u. a. Parathion bei den Sammlerinnen sogenannte Zittertänze hervorruft. Diese Zittertänze können allerdings auch die Folge ganz anderer Ursachen (LINDAUER 1948, v. Frisch 1965, Florey 1954) sein und haben offenbar keinen Kommunikationswert. Nach unseren Beobachtungen von Parathion-vergifteten Sammlerinnen führen diese im Stock heftige zitternde Bewegungen in allen Richtungen aus, die mit der Schwänzelphase des Tanzes im Aussehen eine gewisse Ähnlichkeit zeigen. Die Sammlerinnen können während der Ủbergabe des Sammelgutes die Zitterbewegungen nicht aussetzen, soda $B$ die Futterübergabe trotz großer Bereitschaft der Abnehmerinnen im Stock beinahe unmöglich ist und bisweilen fast eine Stunde dauert. Haben derartig zitternde Sammelbienen letztlich doch ihr Sammelgut übergeben, so verlassen sie unverzüglich den Stock zu einem neuen Sammel tug. Um solche Zitterbewegungen von vornherein zu vermeiden, muBten wir bei unseren Versuchen weit unter den Werten von Schick bleiben. Vorversuche zeigten, daß die einmalige Gabe Parathionvergifteten Zuckerwassers im Wirkstoffverhältnis von $1: 200000$ (5 ppm) noch keine Zitterbewegungen verursachte. Bei einer zweimaligen Giftgabe traten bereits entsprechende Symptome auf.

Bei einer peroralen Vergiftung am Futterplatz muß jedoch berücksichtigt werden, daß die Sammlerinnen das Zuckerwasser im Stock abgeben. Es ist unwahrscheinlich, daß das gesamte aufgenommene Parathion während des Rückfluges bereits vollständig resorbiert wird. Deshalb stellten wir zur Bestimmung der effektiven Giftmengen den folgenden Versuch an :

Bienen, zu einem künstlichen Futterplatz dressiert, werden beim Landen - also vor dem Saugakt - abgefangen und einzeln gekäfigt, um einen gegenseitigen Futteraustausch zu verhindern. In den Abfangkästchen werden sie anschließend aus $0.1 \mathrm{ml}$ Meßpipetten mit vergiftetem Zuckerwasser (5 ppm Parathion) gefüttert. Nach der Vergiftung werden die Pipetten gegen solche mit unvergiftetem Zuckerwasser ausgetauscht, um eine Futterversorgung für die folgenden 72 Beobachtungsstunden zu gewährleisten. In jeder Versuchsreihe werden Kontrollbienen dem gleichen Verfahren unterzogen - mit Ausnahme der Vergiftung — um die natürliche Sterblichkeit in Rechnung stellen zu können.

$A b b .1$ zeigt die Versuchsanordnung. Das Ergebnis ist in $A b b .2$ dargestellt : Perorale Dosen von mehr als 0.03 Gamma Parathion wirken letal. Der Tod tritt innerhalb von 4 Stunden ein, begleitet von Muskelzittern, Taumelbewegungen, heftigen Muskelkrämpfen und letztlich Erbrechen von Futter und Haemolymphe je nach aufgenommener Giftmenge. 32 Bienen, die zwischen 0.005 und 0.03 Gamma Parathion aufgenommen hatten - sie sind in der Abbildung 2 nicht aufgeführt - überlebten die der Vergiftung folgenden 72 Stunden ohne sichtbare Verhaltensänderungen ( $z$. B. unkorrelierte Bewegungen). Sie wurden nach diesen drei Beobachtungstagen wieder freigelassen und sammelten am Futterplatz bis zu 9 Tagen weiter. Das entspricht durchaus der normalen Sammeldauer im Bienenleben.

Wir können aus diesem Ergebnis folgern, da 3 die maximale subletale Dosis von Parathion (= die während des Rückfluges resorbierte Giftmenge), die für die in den folgenden Kapiteln zu besprechenden Verhaltensänderungen in der Entfernungsweisung verantwortlich ist, weniger als 0.03 Gamma beträgt. 


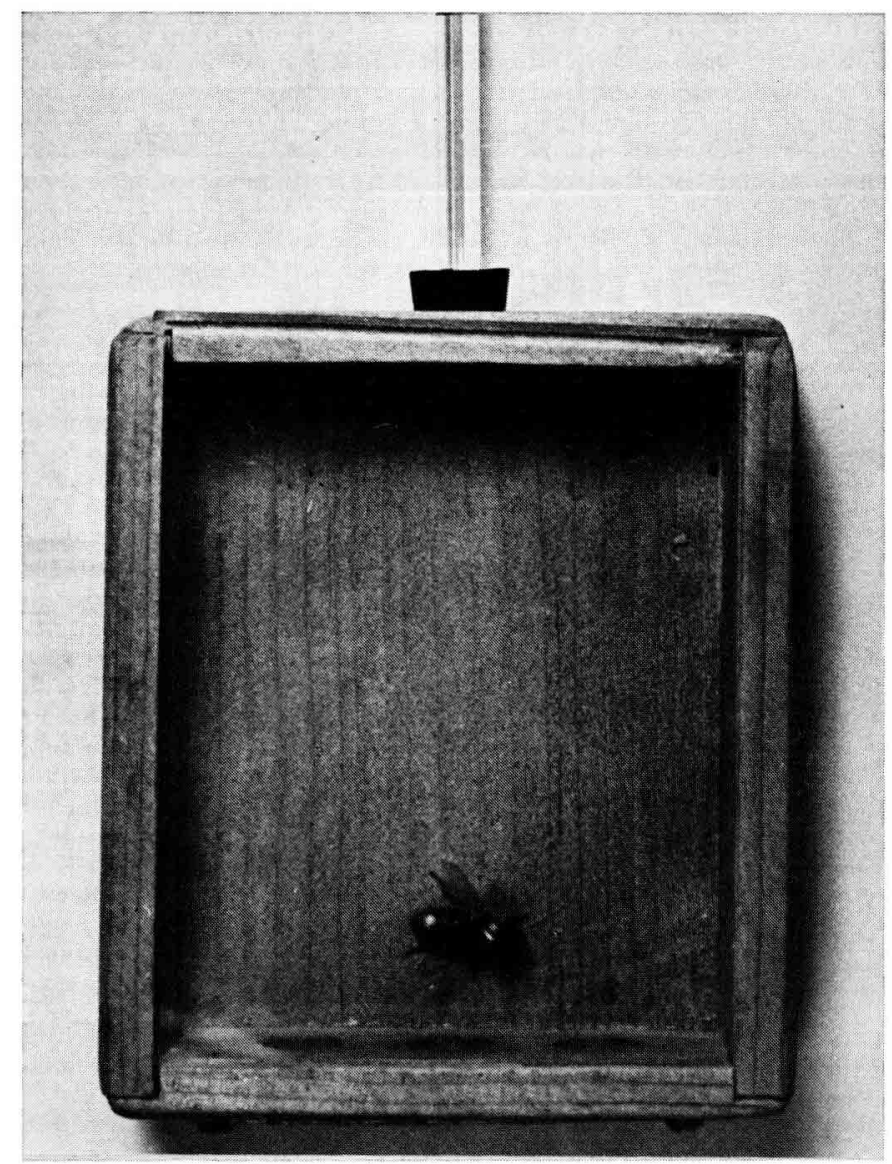

Aвв. 1. - Bestimmung der subletalen Dosis von Parathion. Gehäfigte Biene. Fig. 1. -- Détermination de la dose sublêtale de parathion. Abeille encagée.

\section{III. - ENTFERNUNGSWEISUNG BEI TÄNZEN AUF VERTIKALER WABE}

Bekanntlich gibt das Tempo, in dem ein Werbetanz von der Sammlerin ausgeführt wird, Auskunft über die Entfernung der Futterquelle zum Stock (Zusammenfassung: v. Frisch 1965) : Es nimmt ab bei zunehmender Entfernung. Dabei unterliegt das Tanztempo einer Sammlerin nach jedem Sammel flug zu derselben Futterquelle gewissen individuellen Schwankungen (BRÄUNINGER 1964). Auch das Lebensalter sowie Sammelerfahrung wirken auf das Tanztempo ein (Schweiger 1958, Heran u. Schreindeler 1967).

$A b b .3$ zeigt das Ergebnis eines Versuches, in dem die Bienenschar zu einer Futterquelle in 185 Meter Entfernung dressiert worden war : Unter den 


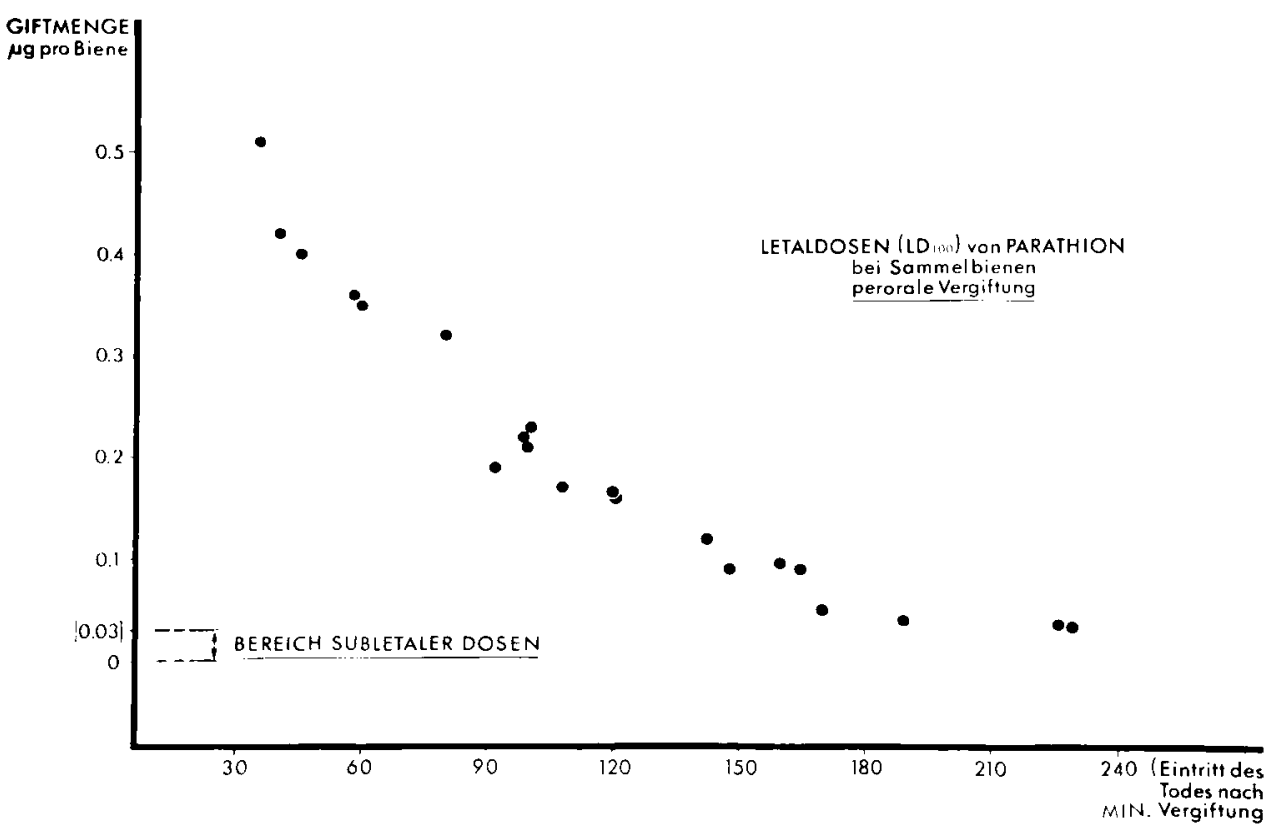

Aвв. 2. - Bestimmung der Subletaldosis von Parathion bei peroraler Vergiftung einzeln gekäfigter Bienen. -: Aufgenommene Giftmenge (Ordinate) einer Biene und die anschließende Uberlebensdauer (Abszisse).

FIg. 2. - Détermination de la dose sublétale de parathion par intoxication par voie orale d'abeilles encagées individuelles.

- dose de poison absorbée (en ordonnées - $\mu g$ par abeille).

En abscisses temps de survie (en minutes) après l'intoxication.

Entre 0,03 et 0 j.g par abeille, domaine des doses sublétales.

gegebenen Versuchsbedingungen benötigen sämtliche Tanzbienen für 10 Tanzumläufe zwischen ca. 18.5 und 23 Sekunden. Nach dem Zeitpunkt der Vergiftung führen die vergifteten Bienen 10 Tanzumläufe in einer signifikant kürzeren Zeit durch, während die Kontrollbienen ihr ursprüngliches Tanztempo beibehalten. Die vergifteten Bienen tanzen schneller und geben damit eine kürzere als die tatsächliche Entfernung für die Futterquelle an (hierzu siehe auch Kapitel V). Das gleiche Ergebnis erhalten wir, wenn die Entfernung zwischen Stock und Futterplatz auf 530 Meter (Abb. 4) oder auf 865 Meter (Abb. 5) vergrößert wird. Dabei vermindert sich das Tanztempo generell entsprechend der größeren Entfernung der Futterquelle.

Bei diesen drei Versuchen umfaßte die gesamte Dressurschar jeweils 14 bis 18 Bienen. Einen Vergleich der individuellen Streuung des Tanztempos von Kontrollbienen und von vergifteten Bienen soll Abb. 6 zeigen : Aus der Dressurschar des in Abbildung 5 dargestellten Versuches wurden die Tanzwerte von vier Bienen herausgenommen - zwei Kontrollbienen und zwei zu 
vergiftende bzw. vergiftete Bienen. Es ist ersichtlich, daß die Breite der individuellen Streuung beider Gruppen nicht voneinander verschieden ist.

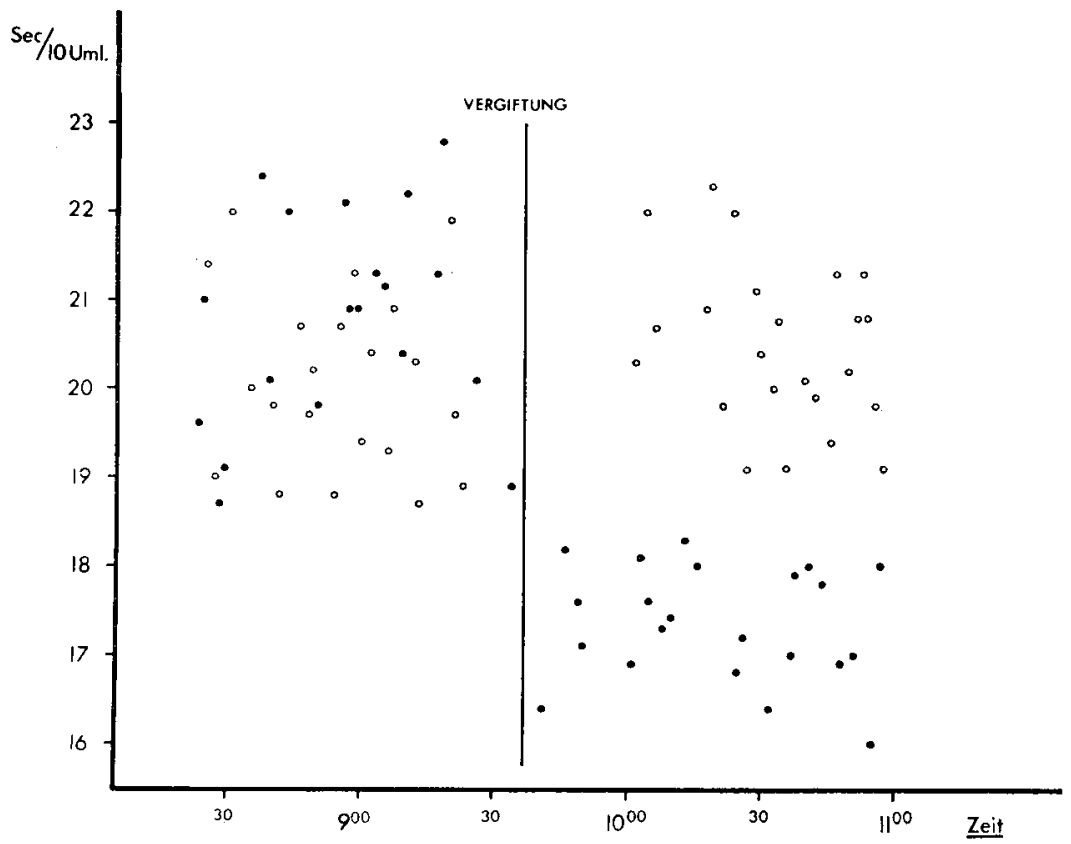

Авв. 3. - Vergleich des Tanztempos auf vertikaler Wabe (Zeit/10 Tanzumläufe) vor und nach der Vergiftung. Futterplatz: 185 Meter Entfernung, Richtung $325^{\circ}$ NNW.

Vor der Vergiftung $=\bigcirc$ Tanzwert einer Kontrollbiene,

- Tanzwert einer zu vergiftenden Biene.

Nach der Vergiftung $=0$ Tanzwert einer Kontrollbiene,

Tanzwert einer vergifteten Biene.

FIG. 3. - Comparaison du tempo de la danse (temps en secondes par 10 tours de danse) sur rayon vertical avant et après intoxication. En abscisse l'heure des observations. Le trait vertical correspond au moment précis de l'intoxications des butineuses. Nourrissement à $185 \mathrm{~m}$. direction $325 \mathrm{NNW}$.

Avant l'intoxication $=O$ tempo d'une abeille témoin,

tempo d'une abeille destinée à être intoxiquée.

Après l'intoxication $=\bigcirc$ tempo d'une abeille témoin,

tempo d'une abeille intoxiquée.

Es ist bekannt, daß Sammelbienen die zwischen Stock und Futterplatz zurückgelegte Strecke aufgrund der verbrauchten Energie messen (BISETZKY 1957, Scholze, Pichler et Heran 1964). Alle Faktoren, die den Flug der Sammlerin direkt behindern oder erleichtern (Heran 1955, Schifferer 1952, v. Frisch u. Lindauer 1955, Heran 1963, Bräuninger 1964) sowie die Futter - und Stocktemperatur (BRÄUNINGER 1964) treten in einer entsprechend veränderten Entfernungsangabe wieder in Erscheinung. Für einen Flug bei Gegenwind wird mehr Energie verbraucht, d. h. die Sammlerin zeigt eine größere Entfernung als die tatsächliche an (BRÄUNINGER 1964, v. Frisch 1965, Heran 1969). Genauso wirken sich niedrige Außentemperaturen oder eine 


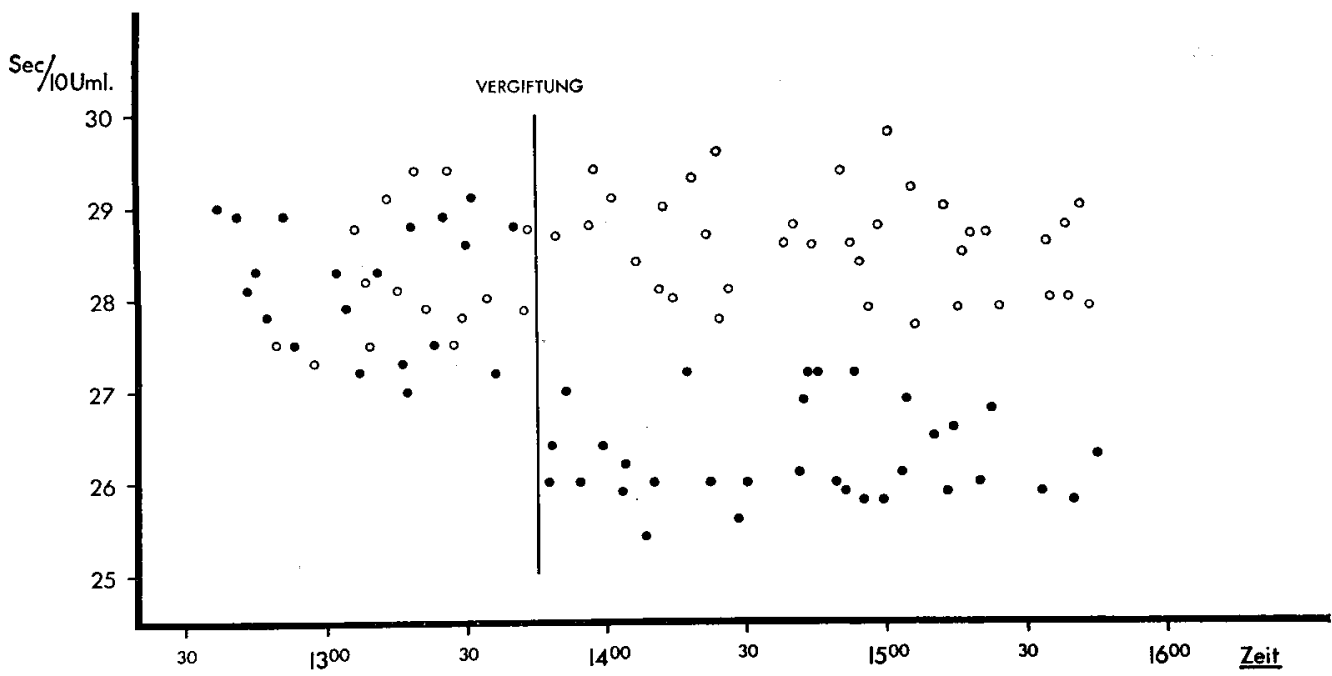

Aв8. 4. - Vergleich des Tanztempos auf vertikaler Wabe (Zeit/10 Tanzumläufe) vor und nach der Vergiftung. Weitere Erklärung : Abbildung 3. Futterplatz : 530 Meter Entfernung, Richtung 337.5० NNW.

FIg. 4. - Comparaison du tempo de la danse (temps en secondes par 10 tours de danse) sur rayon vertical avant et après intoxication.

Autres explications, voir fig. 3.

Nourrissement à $530 \mathrm{~m}$; direction $337^{\circ} 5 \mathrm{NNW}$

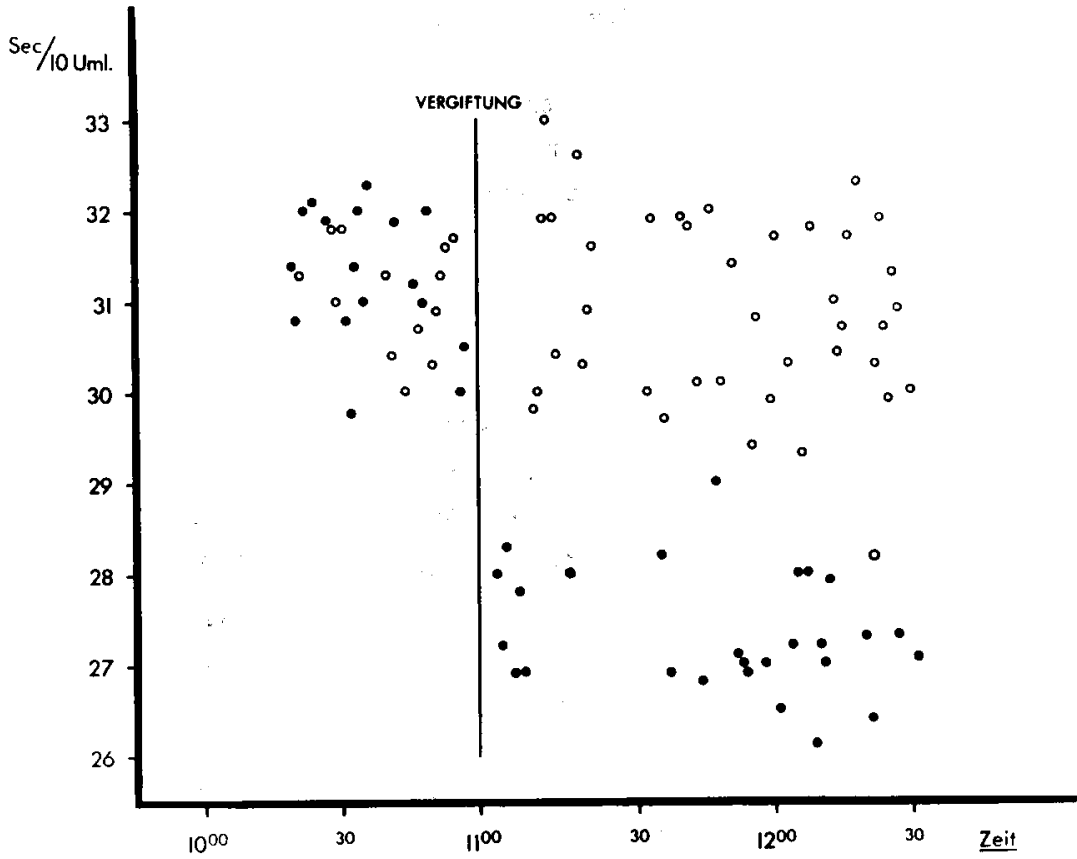

Aвв. 5. - Vergleich des Tanztempos auf vertikaler Wabe (Zeit/10 Tanzumläufe) vor und nach der Vergiftung. Weitere Erklärung : Abbildung 3. Futterplatz : 865 Meter Entfernung, Richtung 301" NW.

Fig. 5. - Comparaison du tempo de la danse (temps en secondes par 10 tours de danse) sur rayon vertical avant et après intoxication.

Autres explications, voir Fig. 3 .

Nourrissement à $865 \mathrm{~m}$; direction $301^{\circ} \mathrm{NW}$. 


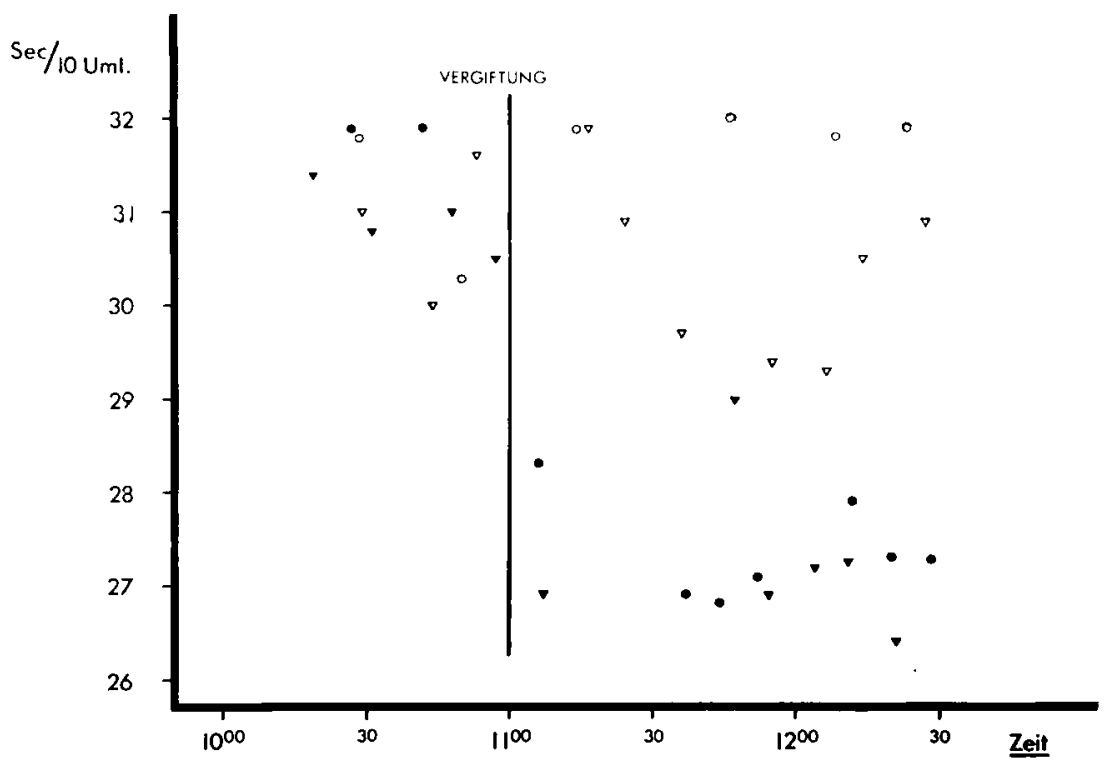

AвB. 6. - Derselbe Versuch wie Abbildung 5. Dargestellt ist das Tanztempo von zwei Kontrollbienen $(\triangle$ und $O$ ) und von zwei zu vergiftenden bzw. vergifteten Bienen ( $\Delta$ und $O$ ).

FIg. 6. - Même expêrience que Fig. 5. On a représenté le tempo de la danse de deux abeilles témoins $(\triangle$ et $\bigcirc)$ et de deux abeilles destinées à être intoxiquées, puis intoxiquées ( $\Delta$ et $\boldsymbol{O})$.

Flugstrecke hangaufwärts auf die Entfernungsweisung aus (HERAN u. Wanke 1952, Heran 1956). Entsprechend wird nach einem Flug hangabwärts oder bei Mitwind aufgrund des niedrigeren Energieaufwandes eine kürzere als die tatsächliche Entfernung im Tanz angezeigt. Wir werden uns mit diesem Problem noch einmal bei der Diskussion unserer Ergebnisse befassen.

Derartige, das Tanztempo verändernde Faktoren wurden in den bisher aufgeführten Versuchen nicht berücksichtigt. Hierbei sollte in jedem Experiment für sich zunächst ein Vergleich angestellt werden zwischen Gruppen, die unter gleichen Witterungsbedingungen sammeln. Ein direkter Vergleich der Tanzwerte für verschiedene Entfernungen ist jedoch nur dann möglich, wenn die Messungen unter identischen Bedingungen gewonnen worden sind.

In der Abb. 7 werden die Mittelwerte der Einzelergebnisse für drei Entfernungen miteinander verglichen, die sämtlich unter folgenden Witterungsbedingungen gewonnen wurden : nahezu Windstille $(<1 \mathrm{~m} / \mathrm{sec})$, wolkenloser Himmel, Außentemperatur während des Versuches $24{ }^{\circ} \mathrm{C}$. Vor der Vergiftung sind die Mittelwerte des Tanztempos von Kontrollbienen und zu vergiftenden Bienen nicht verschieden (Siehe Tabelle I zu Abb. 7), nach der Vergiftung besteht jedoch ein signifikanter Unterschied. Die vergifteten Bienen tanzen schneller als die unvergifteten Kontrollbienen : Für einen Futterplatz in 865 Meter Entfernung beispielsweise geben sie nur etwa 550 Meter an. 


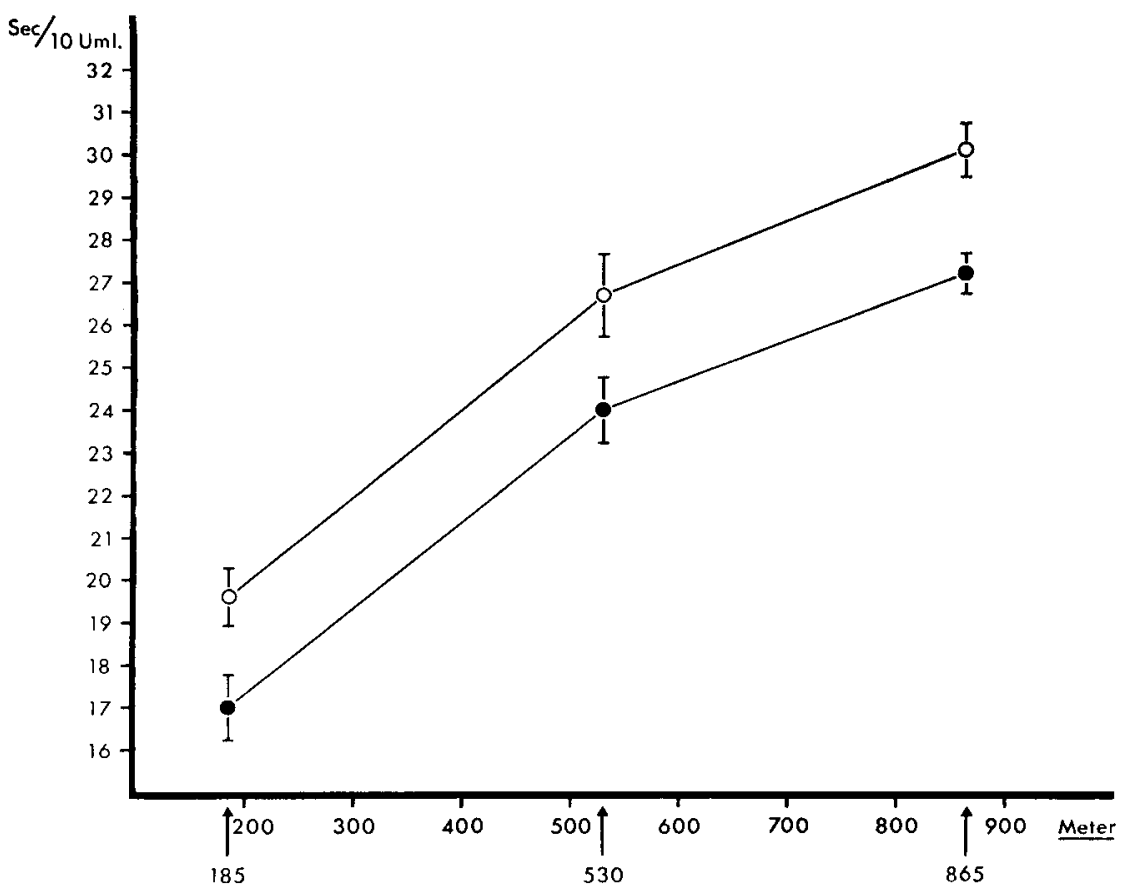

Aвв. 7. - Vergleich der Mittelwerte des Tanztempos auf vertikaler Wabe von Kontrollbienen (O) und vergifteten Bienen (๑) für 3 Futterplätze in 185 Meter, 530 Meter und 865 Meter Entfernung; Gemessen bei nahezu Windstille $(<1 \mathrm{~m} / \mathrm{sec})$ und $24^{\circ} \mathrm{C}$ AuBentemperatur.

Fig. 7. - Comparaison des valeurs moyennes du tempo de la danse sur rayon vertical des abeilles témoins (O) et des abeilles intoxiquées (๑) pour trois distances de nourrissement : 185, 530 et $865 \mathrm{~m}$.

Mesures effectuées par vent à peu près nul ( $<1 \mathrm{~m} \mathrm{sec}$ ) et par une température extérieure de $24^{\circ} \mathrm{C}$.

\section{IV. - ENTFERNUNGSWEISUNG BEI TÄNZEN AUF HORIZONTALER WABE}

Die bisher besprochenen Ergebnisse wurden an Hand von Tanztempomessungen auf der Wabe in Vertikalposition gewonnen. Bekanntlich sind die Sammlerinnen aber auch durchaus in der Lage, ihre Tänze auf horizontalem Untergrund auszuführen (Zusammenfassung : v. FrISCH 1965), beispielsweise auf dem Ab flugbrett (besonders in trachtarmen Zeiten) oder auf der Schwarmtraube, um einen neuen Wohnsitz mitzuteilen (Lindauer 1955).

Es erhebt sich nun die Frage, ob vergiftete Bienen Richtungstänze auf horizontalem Untergrund auch in einem erhöhten Tanztempo ausführen. In früheren Untersuchungen (Schricker u. Stephen 1970, Stephen u. Schricker 1970) stellten wir große Veränderungen in der Richtungsweisung bei vergifteten Tänzerinnen fest : Sammelbienen geben die Richtung zur Futterquelle mit einer Abweichung bis zu 30 Grad bei ihren Tänzen auf vertikaler Wabe an, nachdem sie subletal vergiftet worden waren. Wird ihnen jedoch nur die 
TAB. 1. -- (zu Abbildung 7)

Tanztempo auf vertikaler Wabefür Futterplätze in 185 Meter, 530 Meter und 865 Meter Entfernung zum Stock. Sicherung nach $t$ - Test (Pätad 1943). $\mathrm{n}_{\text {linzel werte }}=$ Zeit pro 10 Tanzumläufe.

Tabl. 1. - (relatif à la figure 7)

Tempo de la danse sur rayon vertical pour des distances de nourrissement de 185,530 et 865 m. Contrôle statistique par test $t$ (PäTAU, 1943). $\mathrm{n}_{\mathrm{E}}$ (valeurs individuelles $=$ temps pour 10 tours de danse).

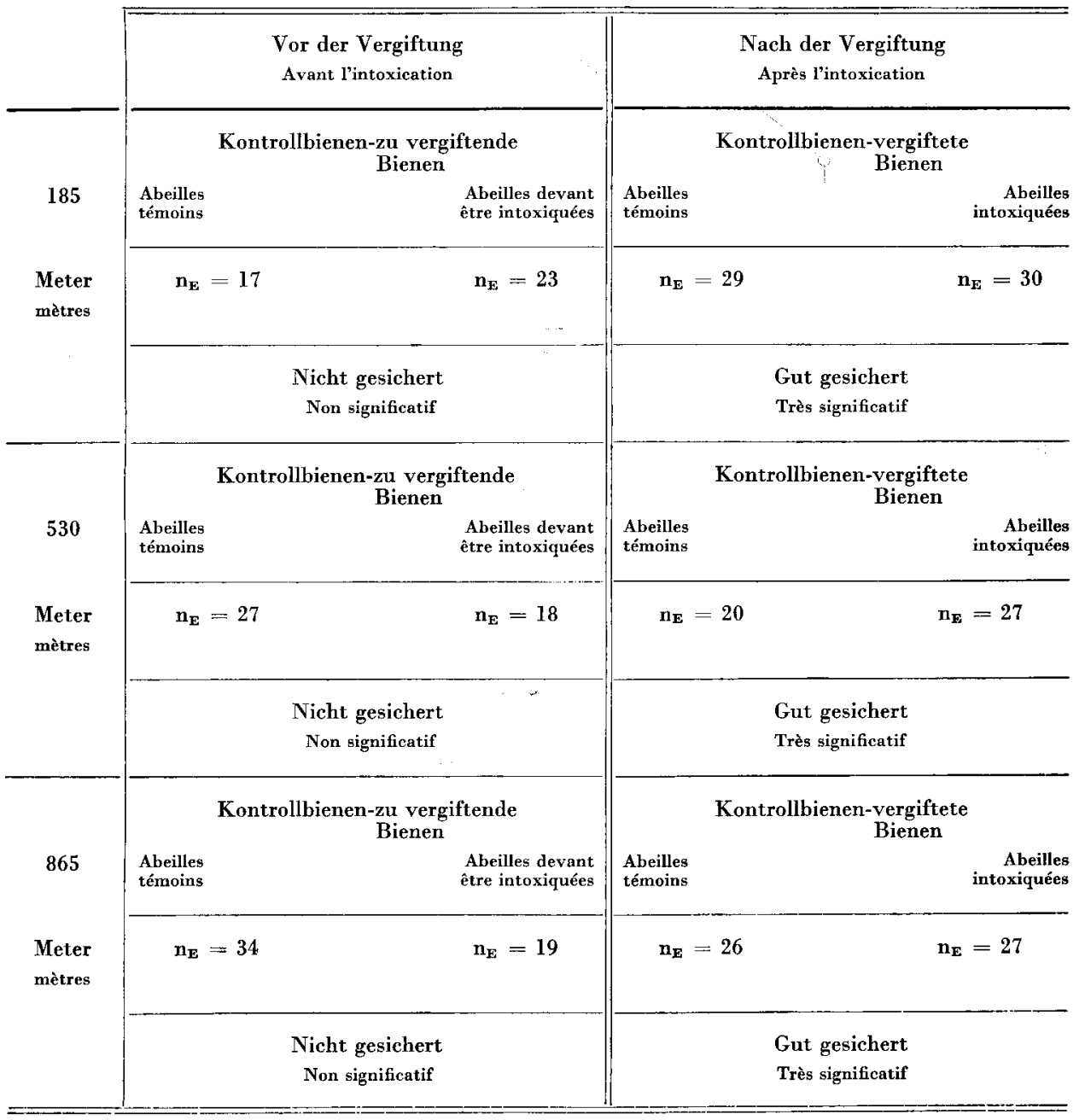


Möglichkeit geboten, Tänze auf horizontalem Untergrund auszuführen, so sind nur unmittelbar nach Vergiftung geringe Abweichungen von der korrekten Richtungsanzeige festzustellen. Durch plötzliches Drehen des Tanzbodens von der Vertikal - zur Horizontalposition (und zurück) kann die Änderung in der Genauigkeit der Richtungsweisung unmittelbar nacheinander festgestellt werden. Aus diesem Ergebnis schlossen wir, daß aufgrund der Vergiftung die Schweresinnesorgane bzw. deren übergeordnete Zentren geschädigt sind : Die Tänzerinnen sind nicht mehr in der Lage, den während des Sammelfluges wahrgenommenen Sonnenwinkel (Winkel zwischen Sonnenazimut, Futterquelle und Stock) zu einem schwerkraftorientierten Tanzwinkel auf der vertikalen Wabe zu transponieren. Dieser Transponierungsvorgang ist bei Tänzen auf horizontaler Unterlage nicht notwendig, d. h. die Richtung kann (bei freier Sicht zur Sonne bzw. dem blauen Himmel) trotz geschädigter Schweresinnesorgane korrekt angezeigt werden.

Was nun die Entfernungsweisung anbetrifft, so wäre es denkbar, daß die vergifteten Sammlerinnen aufgrund des geschädigten Schweresinnes auf der vertikalen Wabe ein größeres Tanztempo vorlegen - sozusagen “ unsicherer » tanzen. Das träfe bei Tänzen auf horizontalem Untergrund nicht zu. Hierzu gibt uns folgender Versuch Auskunft. Zunächst wurde das Tanztempo auf horizontal gelegter Wabe von vergifteten und von unvergifteten Bienen für einen Futterplatz in 865 Meter Entfernung gemessen und miteinander verglichen (Abb. 8) : Die Gegenüberstellung mit dem auf vertikaler Wabe gemessenen Tanztempo für die gleiche Entfernung (Siehe Abbildung 5) ergibt keinen Unterschied. Das wird noch deutlicher un Abb. 9 : : Hier ist wiederum die Entfernungsangabe im Mittel von vergifteten und von unvergifteten Sammlerinnen für drei Futterplätze in unterschiedlicher Entfernung dargestellt. Die Werte wurden ebenfalls unter identischen Außenbedingungen (Siehe Seite 156) gewonnen.

Wir gelangen zu dem Ergebnis, daß Tänzerinnen nach der Vergiftung mit einer subletalen Dosis Parathion auch auf horizontaler Unterlage signifikant (Tabelle II zu Abbildung 9) eine kürzere als die tatsächliche Entfernung (Tanztempo der Kontrollbienen) der Futterquelle angeben. Weiterhin zeigt ein Vergleich von Abbildung 7 mit Abbildung 9, daß es bei der Entfernungsangabe vergifteter Bienen ohne Bedeutung ist, ob die Tanzunterlage sich in vertikaler oder in horizontaler Lage befindet.

\section{V. - INFORMATIONSWERT DER WERBETÄNZE VERGIFTETER SAMMLERINNEN FÜR DIE STOCKGENOSSINNEN}

Die Frage soll erörtert werden, inwieweit die in den Schwänzeltänzen vergifteter Bienen enthaltene Falschinformation von den an der Futterquelle interessierten Stockgenossinnen als solche erkannt wird. In Hinblick auf die 


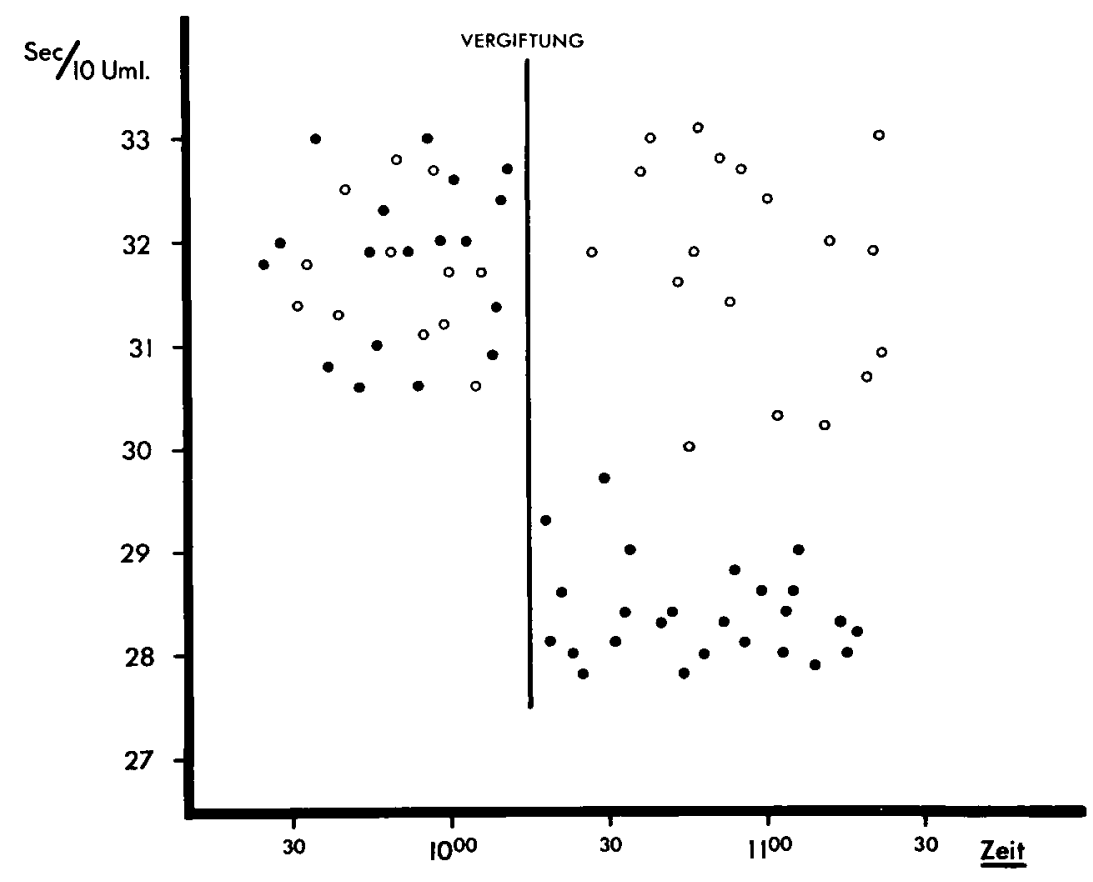

Авв. 8. - Vergleich des Tanztempos auf horizontaler Wabe (Zeit/10 Tanzumläufe) vor und nach der Vergiftung. Zeichenerklärung : Siehe Abbildung 3. Futterplatz : 865 Meter Entfernung, Richtung $301^{\circ} \mathrm{NW}$.

FIG. 8. - Comparaison du tempo de la danse (temps en secondes par 10 tours de danse sur rayon horizontal avant et après l'intoxication.

Explications des signes : voir figure 3.

Distance de nourrissement : $865 \mathrm{~m}$. Direction $301^{\circ} \mathrm{NW}$.

Richtungsweisung trifft das nicht zu (Stephen u. Schricker 1970) : Neulinge richten sich nach den Tanzwinkeln und werden entsprechend in der Richtung fehlgeleitet. Aus diesen Versuchen kann allerdings aufgrund der angewandten Methode (Fächerversuch) keine Aussage darüber gemacht werden, ob ebenfalls die verfälschte Entfernungsweisung von den Neulingen befolgt und damit der Futterplatz in einer kürzeren Entfernung gesucht wird. Hierzu wurde der folgende Stufenversuch durchgeführt.

Zur Methode : Ein Volk wird in ein vom Heimatgebiet etwa 30 Kilometer entfernt gelegenes, den Bienen fremdes Gelände transportiert. Dort wird eine individuell markierte Schar in der üblichen Weise zu einem Futterplatz in 755 Meter Entfernung dressiert und darauf - wie auch an den beiden folgenden Tagen - für 2 Stunden mit verdünnter Zuckerlösung gefüttert, damit keine Neulinge geworben werden. Der Futterplatz bleibt ausschließlich der Dressurschar bekannt. Nach der jeweiligen Fütterung wird das Flugloch des Stockes geschlossen, und alle übrigen heimkehrenden Bienen werden abgefangen und gekäfigt. Sie werden dem Volk nicht mehr zugegeben. An- 


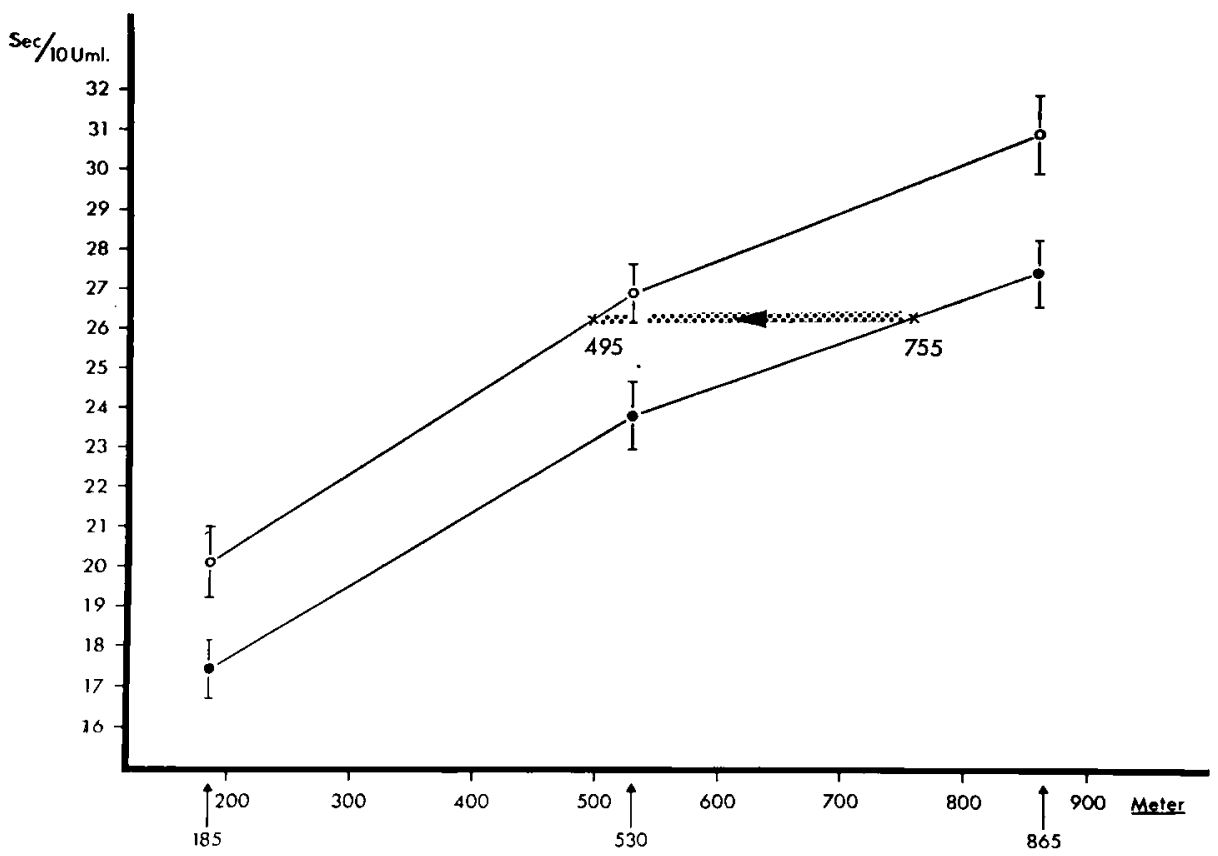

AвB. 9. - Vergleich der Mittelwerte des Tanztempos auf horizontaler Wabe von Kontrollbienen (O) und von vergifteten Bienen (O) für 3 Futterplätze in 185 Meter, 530 Meter und 865 Meter Entfernung; Gemessen bei nahezu Windstille $(<1 \mathrm{~m} / \mathrm{sec})$ und $24^{\circ} \mathrm{C}$ Außentemperatur.

Vergiftete Tänzerinnen geben für eine Futterquelle in 755 Meter Entfernung nur 495 Meter an.

FIG. 9. - Comparaison des valeurs moyennes du tempo de la danse sur rayon horizontal. Abeilles témoins $(O)$ et abeilles intoxiquées (O) pour trois distances de nourrissement : 185,530 et $865 \mathrm{~m}$. Mesures effectuées par vent à peu près nul $\left(<1 \mathrm{~m} / \mathrm{sec}\right.$.) et par une température extérieure de $24^{\circ} \mathrm{C}$.

Les danseuses intoxiquées n'indiquent pour une source de nourriture située à $755 \mathrm{~m}$ qu'une distance de $495 \mathrm{~m}$.

schließend wird das Volk in sein Heimatgebiet zurücktransportiert. Am 4. Tag werden zu Beginn des kritischen Versuches Duftplatten in 495 Meter und 645 Meter Entfernung ausgelegt. Diese Platten sind lediglich mit dem Dressurduft versehen, der in dem am Futterplatz gebotenen (diesmal 2-molaren) Zuckerwasser enthalten ist. An jeder Duftplatte ist ein Beobachter postiert. Die von den dressierten Tänzerinnen geworbenen Neulinge “ verknüpfen » den Dressurduft, den sie von der Tänzerin in einer Futterprobe gereicht bekommen haben, fest mit der Futterquelle. Sie lassen sich auf ihrem Weg zum Futterplatz an einer der Duftplatten nieder, in deren Duftzone sie gelangt waren. Dort werden sie vom Beobachter gewertet.

Aus den vorher dargelegten Gründen, daß nämlich Neulinge durch die veränderte Richtungsweisung vergifteter Tänzerinnen entsprechend fehlgeleitet werden, konnten wir den Versuch nicht in der klassischen Form des Stufenversuches ausführen. Er mußte vielmehr als ein kombinierter Stufen - Fächerversuch vorbereitet werden : Deshalb wurden Duftplatten an den 
TABEL. 2. - (zu Abbildung 9)

Tanztempo auf horizontaler Wabe für Futterplätze in 185 Meter, 530 Meter und 865 Meter Entfernung

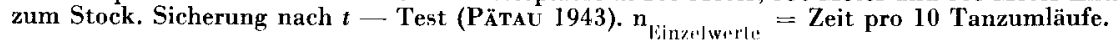

TABL. 2. - (relatif à la figure 9)

Tempo de la danse sur rayon horizontal pour des distances de nourrissement de $185 \mathrm{~m}, 530 \mathrm{~m}$ et $865 \mathrm{~m}$. Contrôle statistique par test $t\left(\mathrm{P}_{\mathrm{A} T A U}, 1943\right) . \mathrm{n}_{1:}$ (valeurs individuelles $=$ temps pour 10 tours de danse).

\begin{tabular}{|c|c|c|c|c|c|}
\hline & \multicolumn{2}{|c|}{$\begin{array}{l}\text { Vor der Vergiftung } \\
\text { Avant l'intoxication }\end{array}$} & \multicolumn{3}{|c|}{$\begin{array}{l}\text { Nach der Vergiftung } \\
\text { Après l'intoxication }\end{array}$} \\
\hline & \multicolumn{2}{|c|}{$\begin{array}{c}\text { Kontrollbienen-zu vergiftende } \\
\text { Bienen }\end{array}$} & \multicolumn{3}{|c|}{$\begin{array}{c}\text { Kontrollbienen-vergiftete } \\
\text { Bienen }\end{array}$} \\
\hline 185 & $\begin{array}{l}\text { Abeilles } \\
\text { témoins }\end{array}$ & $\begin{array}{l}\text { Abeilles devant } \\
\text { être intoxiquées }\end{array}$ & $\begin{array}{l}\text { Abeilles } \\
\text { témoins }\end{array}$ & & $\begin{array}{r}\text { Abeilles } \\
\text { intoxiqquées }\end{array}$ \\
\hline \multirow{3}{*}{$\begin{array}{l}\text { Meter } \\
\text { mètres }\end{array}$} & $\mathbf{n}_{\mathbf{E}}=21$ & $\mathbf{n}_{\mathbf{E}}=\mathbf{3 4}$ & $n_{\mathbf{E}}=46$ & & $\mathbf{n}_{\mathbf{E}}=40$ \\
\hline & \multicolumn{2}{|c|}{$\begin{array}{l}\text { Nicht gesichert } \\
\text { Non significatif }\end{array}$} & \multicolumn{3}{|c|}{$\begin{array}{l}\text { Gut gesichert } \\
\text { Très significatif }\end{array}$} \\
\hline & \multicolumn{2}{|c|}{$\begin{array}{c}\text { Kontrollbienen-zu vergiftende } \\
\text { Bienen }\end{array}$} & \multicolumn{3}{|c|}{$\begin{array}{c}\text { Kontrollbienen-vergiftete } \\
\text { Bienen }\end{array}$} \\
\hline 530 & $\begin{array}{l}\text { Abeilles } \\
\text { témoins }\end{array}$ & $\begin{array}{l}\text { Abeilles devant } \\
\text { être intoxiquées }\end{array}$ & $\begin{array}{l}\text { Abeilles } \\
\text { témoins }\end{array}$ & & $\begin{array}{r}\text { Abeilles } \\
\text { intoxiquées }\end{array}$ \\
\hline \multirow{2}{*}{$\begin{array}{l}\text { Meter } \\
\text { mètres }\end{array}$} & $\mathrm{n}_{\mathbf{E}}=24$ & $\mathbf{n}_{E}=16$ & $\mathrm{n}_{\mathrm{E}}=16$ & & $\mathbf{n}_{\mathbf{E}}=20$ \\
\hline & \multicolumn{2}{|c|}{$\begin{array}{l}\text { Nicht gesichert } \\
\text { Non significatif }\end{array}$} & \multicolumn{3}{|c|}{$\begin{array}{l}\text { Gut gesichert } \\
\text { Très significatif }\end{array}$} \\
\hline & \multicolumn{2}{|c|}{$\begin{array}{c}\text { Kontrollbienen-zu vergiftende } \\
\text { Bienen }\end{array}$} & \multicolumn{3}{|c|}{$\begin{array}{c}\text { Kontrollbienen-vergiftete } \\
\text { Bienen }\end{array}$} \\
\hline 865 & $\begin{array}{l}\text { Abeilles } \\
\text { témoins }\end{array}$ & $\begin{array}{l}\text { Abeilles devant } \\
\text { être intoxiquées }\end{array}$ & $\begin{array}{l}\text { Abeilles } \\
\text { témoins }\end{array}$ & & $\begin{array}{r}\text { Abeilles } \\
\text { intoxiquées }\end{array}$ \\
\hline \multirow[t]{2}{*}{$\begin{array}{l}\text { Meter } \\
\text { mètres }\end{array}$} & $\mathbf{n}_{\mathbf{x}}=34$ & $\mathbf{n}_{E}=26$ & $\mathbf{n}_{\mathbf{E}}=20$ & & $\mathbf{n}_{\mathbf{E}}=25$ \\
\hline & & & \multicolumn{3}{|c|}{$\begin{array}{l}\text { Gut gesichert } \\
\text { Très significatif }\end{array}$} \\
\hline
\end{tabular}


angegebenen Punkten nicht nur entlang der Verbindungslinie zwischen Stock und Futterplatz ausgelegt, sondern ebenfalls seitlich der Verbindungslinie.

Das Ergebnis ist in Abb. 10 dargestellt : Vor dem Zeitpunkt der Vergiftung werden in der ersten (A) und in der zweiten (B) halben Stunde die meisten von den Dressurbienen geworbenen Neulinge an der dem Futterplatz in Richtung und Entfernung nächstgelegenen Duftplatte (bei 645 Meter) gezählt. Diese Werte stellen die Kontrolle dar. Nach der Vergiftung der gesamten Dressurschar am Futterplatz ändert sich das Verteilungsbild : In den nächsten beiden halben Stunden (C und D) sucht die Mehrzahl der Neulinge an den Duftplatten in 495 Meter Entfernung. Die Dressurbienen sammeln dabei weiter am Futterplatz. Vergleicht man dieses Ergebnis mit dem in Abbildung 9 dargestellten (graupunktierte Hinweislinie), so fällt auf, daß auch dort vergiftete Bienen eine Entfernung von 750 Metern in einem Tanztempo anzeigen, das dem unvergifteter Bienen für ca. 500 Meter entspricht.

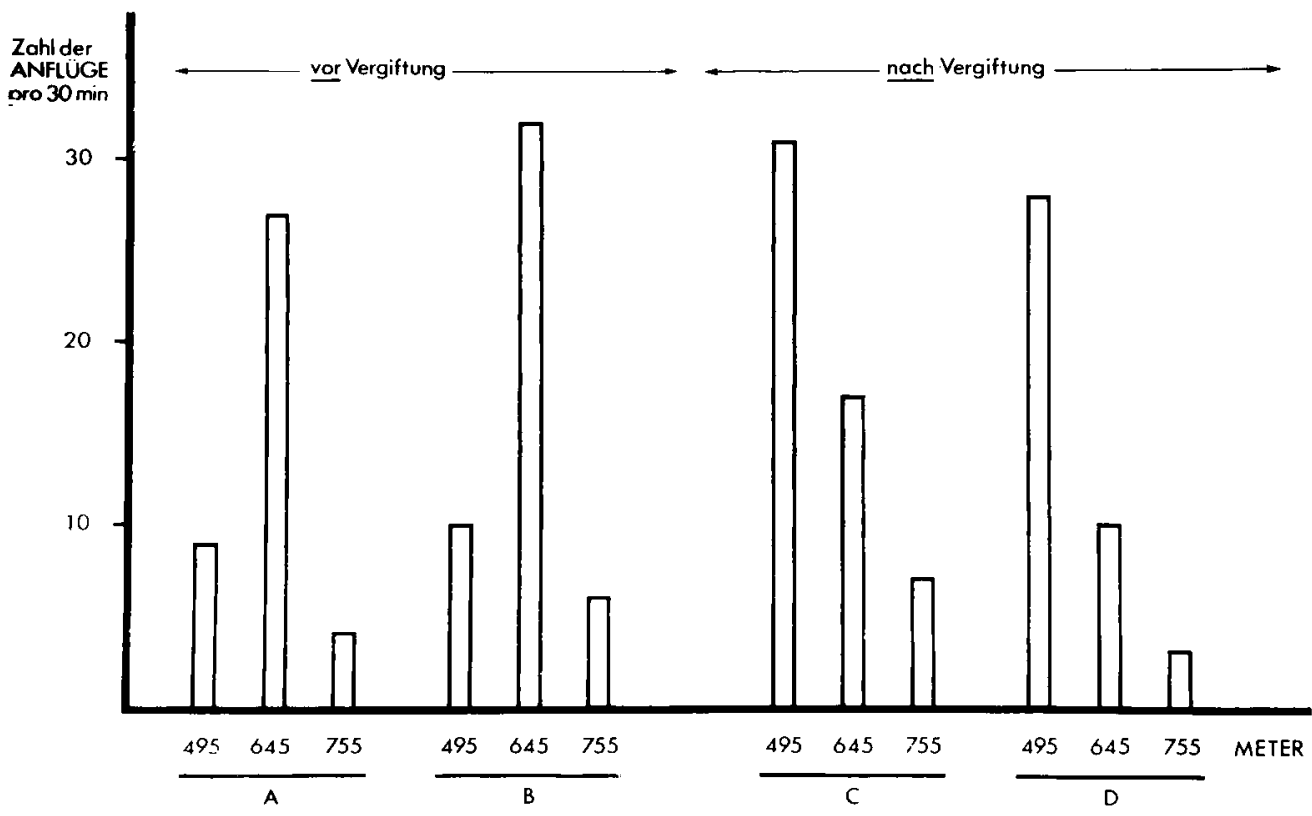

Авв. 10. - Kombinierter Stufen - Fächerversuch. Futterplatz in 755 Meter Entfernung und $347^{\circ}$ NNW. Die Anzahl der Neulinge ist in der Abbildung für jeweils alle Platten einer Entfernungsstufe zusammengefaßt, da bei diesem Versuch nur die Entfernungsweisung interessieren soll. Weitere Erklärung : Siehe Text.

Fic. 10. - Expérience combinée distance et direction. Nourrissement à $755 m$ de distance. Direction $347^{\circ} \mathrm{NNW}$ Sur la figure le nombre des novices (en ordonnées) concerne à chaque fois toutes les plaques qui se trouvent à une distance donnée car, dans cette expérience, seule l'indication de la distance nous intéresse. Explications complémentaires dans le texte.

Seit einigen Jahren werden von einer amerikanischen Forschergruppe (Zusammenfassung : Wenner 1971) Zweifel an der Deutung des Schwänzeltanzes erhoben, wie sie Karl v. Frisch getroffen hat und wie sie seither in 
zahlreichen Untersuchungen erneut belegt worden ist (Zusammenfassung : Esch u. Bastian 1970, Lindauer 1971). Die Gruppe Wenner stellt dabei den Informationswert des Schwänzeltanzes in Hinblick auf Entfernungsweisung und Richtungsweisung in Frage und schließt aus eigenen Ergebnissen, daß Neulinge eine Futterquelle nur aufgrund des Duftes lokalisieren, den sie an den heimkehrenden Sammlerinnen wahrgenommen haben oder durch den eine Futterquelle von dort sammelnden Bienen markiert wird (Sekret der Nassanoff'schen Drüse). Das Ergebnis unseres Stufen - Fächerversuches widerspricht wiederum eindeutig dieser Annahme : Die einzigen Variablen innerhalb dieses Experiments sind einmal das veränderte Tanztempo der Dressurbienen nach deren Vergiftung, weiterhin die veränderten Tanzwinkel, die auch hier berücksichtigt worden sind. Sie sollen an dieser Stelle nicht erneut diskutiert werden (Siehe : Stephen u. Schricker 1970).

Zusammenfassend kann gesagt werden : Neulinge, die von vergifteten Tänzerinnen Information über die Lage einer Futterquelle erhalten, werden sowohl in Entfernung als auch in der Richtung fehlgeleitet.

Von weiterem Interesse ist die Frage, ob der Vergiftungsprozeß sich auch bei den betroffenen Sammlerinnen bemerkbar macht, beispielsweise in der eigenen Entfernungsmessung. Es wurde bereits betont, daß Dressurbienen auch nach einer Vergiftung immer wieder sicher zum Futterplatz zurückkehren, obwohl sie eine kürzere als die tatsächliche Entfernung in ihren Werbetänzen ausdrücken. Orientieren sie sich nur noch nach Landmarken? Diese Frage soll im folgenden Kapitel behandelt werden.

\section{VI. - EINWIRKUNG DER VERGIFTUNG AUF DEN VORGANG DER ENTFERNUNGSMESSUNG}

Im Kapitel III wurde bereits diskutiert, daß die Entfernung zu einer Futterquelle aufgrund der während des Hinfluges verbrauchten Energie gemessen wird. Subletal mit Parathion vergiftete Bienen erhöhen ihre Eigenfluggeschwindigkeit um etwa 10 Prozent (Schricker u. Stephen 1970). Diese Tatsache läßt ebenso wie das erhöhte Tanztempo eine durch das Gift verursachte Hyperaktivität der Bienen vermuten. Auf den möglichen Wirkmechanismus wird im Kapitel VII eingegangen werden.

Diese Vermutung wurde in Laufversuchen getestet, bei denen die positive Phototaxis der Testbienen zu einer Lichtquelle hin ausgenützt wird.

Zur Methode : Die Versuchsbienen durchlaufen einzeln auf ihrem Weg zur Lichtquelle in einem Glasrohr die Meßstrecke von 50 Zentimetern. Die Läufe werden mit jedem Tier mehrmals wiederholt. Anschließend wird die Biene mit einer vergifteten Zuckerlösung aus einer Meßpipette gefüttert und darauf wieder in den Versuch genommen. Die Beleuchtungsstärke der konstant eingestellten Lichtquelle wird durch Graufilter verändert. 
In Abb. 11 ist das Ergebnis dieser Versuchsreihe dargestellt : Die pro Sekunde zurückgelegte Strecke (in Zentimetern) vor und nach der Vergiftung. Dieselben Testbienen erhöhen ihre Laufgeschwindigkeit nach der Vergiftung signifikant um etwa 10 Prozent.

\begin{tabular}{|c|c|c|c|c|}
\hline \multirow{2}{*}{} & \multicolumn{4}{|c|}{ BELEUCHTUNGSSTÄRKE ( Lux) } \\
\cline { 2 - 5 } & 3.5 & 154 & 1280 & 8300 \\
\hline \multirow{3}{*}{$\begin{array}{r}\text { Normal } \\
n\end{array}$} & $7.4 \pm 0.5$ & $7.6 \pm 0.6$ & $75 \pm 0.5$ & $7.7 \pm 0.6$ \\
\cline { 2 - 5 } & 75 & 75 & 75 & 75 \\
\hline $\begin{array}{r}\text { Vergiftet } \\
n\end{array}$ & $8.2 \pm 0.4$ & $8.3 \pm 0.7$ & $8.3 \pm 0.6$ & $8.3 \pm 0.7$ \\
\cline { 2 - 5 } & 75 & 75 & 75 & 75 \\
\hline Sicherung & 0.0015 & $<0.0002$ & $<0.0005$ & $<0.0005$ \\
\hline
\end{tabular}

Авв. 11. - Vergleich der Laufgeschwindigkeit ( $\mathrm{cm} / \mathrm{sec}$ ) von Bienen vor und nach der peroralen Vergiftung mit einer Subletaldosis Parathion gegen eine Lichiquelle bei verschiedenen Beleuchtungsstärken.

Fig. 11. - Comparaison de la vitesse de marche (en $\mathrm{cm} / \mathrm{sec})$ d'abeilles avant et après l'intoxication par voie orale avec une dose sublétale de parathion. Marche vers une source lumineuse avec différentes intensitês (en Lux).

Man kann sich nun vorstellen, daß sich das Tanztempo erhöht, wenn jeder Tanzumlauf aufgrund der erhöhten Laufgeschwindigkeit in einer etwas kürzeren Zeit beendet wird. Diese Deutung kann jedoch nicht befriedigen, denn sie erklärt nicht, warum vergiftete Tänzerinnen trotzdem für verschiedene Entfernungen ein - wenn auch gegenüber unvergifteten Bienen erhöhtes entsprechend unterschiedliches Tanztempo vorlegen, wie aus den Abbildungen 7 und $9 \mathrm{zu}$ ersehen ist. Auch vergiftete Tänzerinnen sind demnach noch relativ über die Entfernung der Futterquelle informiert.

Eine Hyperaktivität, wie wir sie in der Form erhöhter Flug — und Laufgeschwindigkeit diskutiert haben, ist mit einem erhöhten Energieverbrauch verbunden. Diese Tatsache scheint den Ergebnissen zu widersprechen, die Bisetzixy (1957) und Scholze, Pichler u. Heran (1964) gewonnen haben : Erhöhter Energieverbrauch bedeutet die Angabe einer größeren Entfernung und wird in entsprechend verlangsamtem Tanztempo ausgedrückt. Wir wollen zunächst von der Annahme ausgehen, daß vergiftete Tänzerinnen noch über die für den Flug zur Futterquelle benötigte Energie informiert sind. Dann müßten sie aufgrund des erhöhten Energieverbrauches ein langsameres Tanztempo vorlegen. Es wäre nun denkbar, daß das in unseren Experimenten festgestellte erhöhte Tanztempo zustande kommt, indem die größere Laufgeschwindigkeit dem von den Bienen beabsichtigten Tanztempo sozusagen entgegenwirkt und es “ beschleunigend » verfälscht. Dazu sollte ein Versuch 
Auskunft geben können, in dem die durch Vergiftung veränderte Entfernungsangabe nicht in einem entsprechenden Tanztempo, sondern in einer anderen Tanzform ausgedrückt wird.

Den verschiedenen Rassen der Honigbiene ist gemeinsam, daß sie für eine nahe gelegene Futterquelle mit sogenannten Rundtänzen werben, die nur Information über die Entfernung enthalten. Futterplätze in größerer Entfernung werden richtungs - und entfernungsgemäß durch Schwänzeltänze mitgeteilt. Der Wechsel zwischen den beiden Tanzformen liegt für die verschiedenen Rassen bei unterschiedlichen Entfernungen und ist fließend. Außerdem kommt bei einigen Rassen der sogenannte Sicheltanz als dritte Tanzform zwischen Rund - und Schwänzeltanz vor (Восн 1957). Bei unseren Versuchsbienen der Kärntner Rasse liegt der Übergangsbereich zwischen knapp 20 Meter und 85 Meter.

Zur Methode : Wir wandern mit einer individuell markierten Dressurschar und dem Futterplatz so weit, bis im Stock die ersten gerichteten Schwänzelbewegungen bei den Tänzen zu erkennen sind. Das tritt in diesem Versuch bei 38 Meter ein. Die Dressurschar darf eine weitere halbe Stunde am Futterplatz sammeln, während ihr Tanzverhalten im Stock beobachtet wird. Darauf werden alle (acht) Bienen oral vergiftet.

Nach unserer oben angeführten Annahme wäre zu erwarten, daß die Bienen nach der Vergiftung aufgrund des durch die größere Fluggeschwindigkeit erhöhten Energieverbrauches vermehrt Schwänzelbewegungen oder sogar Schwänzeltänze ausführen würden. Das traf allerdings nicht zu. Die Frage bleibt offen, ob es an der Methode gelegen hat, weil beispielsweise der Entfernungsbereich bis zur eindeutigen Ausführung von Schwänzeltänzen noch zu groß war. Oder ist der Unterschied in der verbrauchten Energie zu gering (die Flugzeit beträgt zwischen 5 und 10 Sekunden), um eindeutig mitgeteilt zu werden? Allein hieraus könnte man sich den “ breiten » Übergangsbereich vom Rundtanz zum Schwänzeltanz generell erklären.

Werner (1954) hat im Rahmen von Untersuchungen zum Einfluß stoffwechselfördernder (Thyroxin, Adrenalin) und stoffwechselhemmender (Chinin) Pharmaka auf das Tanztempo einen ähnlichen Versuch ausgeführt. Obwohl die genannten Pharmaka das Tanztempo beein flussen, konnte auch in diesem Fall kein Wechsel von Rundtänzen zu Schwänzeltänzen (bzw. umgekehrt) festgestellt werden. Die Reaktion der Neulinge auf das veränderte Tanztempo wurde nicht untersucht.

Die Klärung dieses Problems mußte auf andere Weise gesucht werden. Dazu hatten wir uns folgenden Versuch überlegt : Abb. 12.

Zur Methode : Eine Gruppe von 12 individuell markierten Bienen wird zu einem Futterplatz in 605 Meter Entfernung $\left(330^{\circ} \mathrm{NNW}\right)$ vom Stock dressiert und dort eine Stunde lang mit 2-molarer Zuckerlösung gefüttert, die mit 


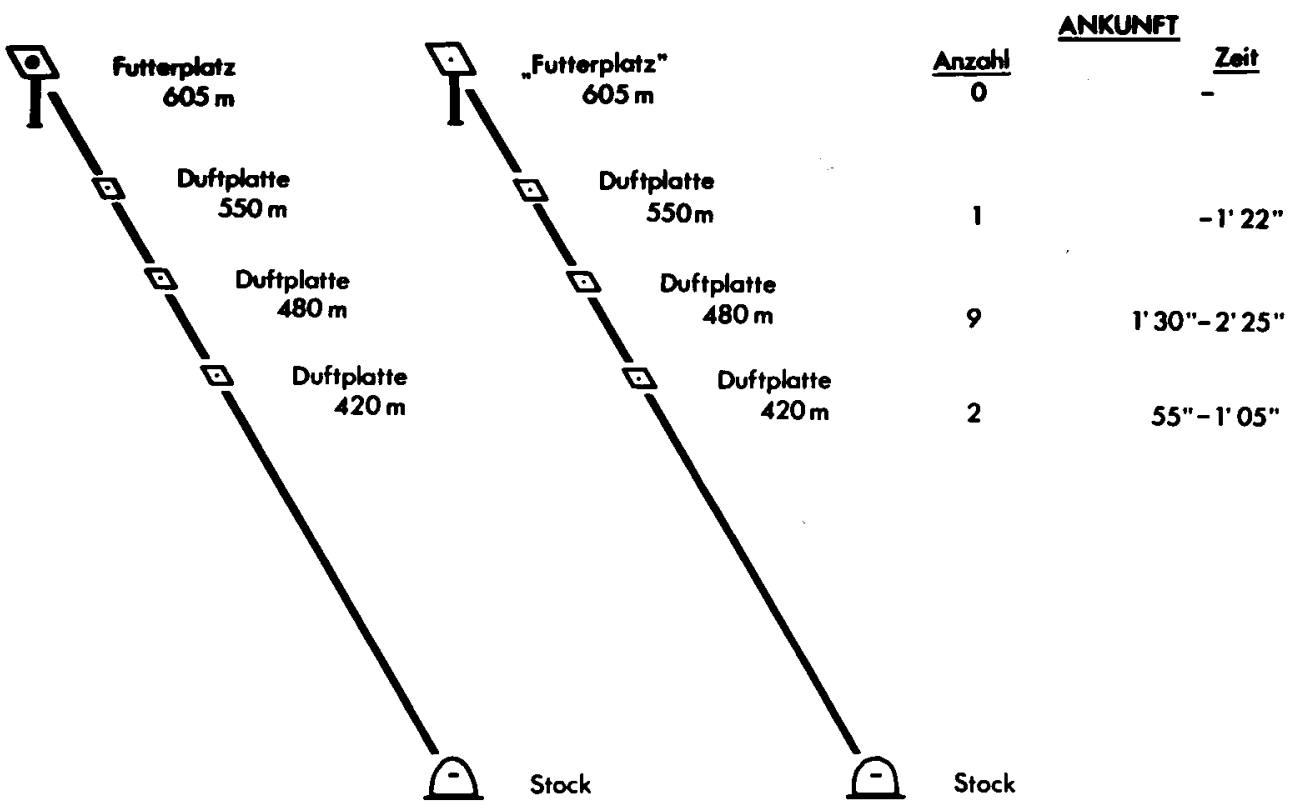

A: Dressurgelände $\frac{\text { Versetzung (12 Bienen) }}{\text { nach Vergiftung }}$ B: unbekanntes Gelände

Aв8. 12. - Stufenversuch nach Versetzung in unbekanntes Gelände (B) mil einer vergifteten Schar, die auf einen Futterplatz in 605 Meter Entfernung und $330^{\circ} \mathrm{NNW}$ dressiert ist $(A)$. Während des Versuches herrschte sehr schwacher Wind $(>1 \mathrm{~m} / \mathrm{sec})$ aus West. Weitere Erklärung : Siehe Text.

Fıg. 12. - Expérience de déplacement d'un groupe d'abeilles intoxiquées vers un terrain inconnu (B). Ces abeilles étaient dressées à venir sur un nourrisseur situé à $605 \mathrm{~m}$ de distance par $330^{\circ} \mathrm{NNW}$ (A). Pendant l'expérience règne un très faible vent d'ouest (>1 m/sec). Explications complémentaires dans le texte.

Geraniumduft versehen ist. Am darauffolgenden Tag werden während der zweistündigen Fütterung entlang der Fluglinie (Abbildung $12: A$ ) Stock Futterplatz mit Geraniumduft versehene Platten in 420 Meter, 480 Meter und 550 Meter ausgelegt, die von den Dressurbienen auf ihrem Weg zum Futterplatz bzw. zum Stock immer wieder über flogen werden. Ein Absitzen der Dressurbienen an den Platten wird nicht beobachtet : sie sind offenbar gut auf den Futterplatz eingeflogen. Am dritten Versuchstag wird zunächst genauso verfahren wie am zweiten. Gegen Ende der Fütterungszeit werden alle 12 Bienen am Futterplatz in der üblichen Weise peroral vergiftet, abgefangen und gekäfigt. Die vergiftete Schar wird anschließend im lichtdichten Kästchen zum Stock gebracht und dort in das Stockinnere hineingeschleust. Der verschlossene Stock wird darauf in ein den Bienen unbekanntes Gelände versetzt (ca. 6 Kilometer vom Heimatstand entfernt). Dort (Abbildung $12: B$ ) 
ist bereits eine identische Versuchsanordnung vorbereitet : Ein (c Futterplatz ) in 605 Meter $\left(330^{\circ} \mathrm{NNW}\right)$ und Duftplatten bei 420 Meter, 480 Meter und 550 Meter. “ Futterplatz 》 und Duftplatten werden jeweils von einem Beobachter bewacht. Vor dem Öffnen des Flugloches wird etwas Geraniumduft in den Stock geblasen, der die Dressubienen am Flugloch erscheinen läßt. Nach dem Öffnen verlassen die Dressurbienen sofort den Stock und fliegen nach wenigen Orientierungsschleifen in Stocknähe in der Dressurrichtung ab (Eine genaue Messung dieser Abflugrichtung steht aus und wird im Rahmen von Untersuchungen zur optischen Orientierungsleistung vergifteter Bienen vorgenommen. Sie ist an dieser Stelle nicht von unmittelbarem Interesse). Die Ankunft jeder Dressurbiene an einem der Beobachtungspunkte wird registriert. Das Tier wird sofort in Alkohol getötet, um eine eventuelle Anlokkung anderer Versuchstiere zu verhindern.

Als Ergebnis dieses Versetzungsversuches stellen wir fest :

1. Alle 12 Bienen haben einen Sammelflug in der Dressurrichtung ausgeführt.

2. Keine der Bienen wurde am “ Futterplatz » in 605 Meter registriert, der der Dressurentfernung entspricht.

3. Der Zeitraum zwischen Öffnen des Stockes und der Landung an einem der Beobachtungspunkte liegt zwischen 55 Sekunden und knapp 2.5 Minuten.

Da die vergifteten Sammlerinnen den Sammelflug in unbekanntem Gelände ausführen mußten, konnten sie (a) die Dressurrichtung nur mit Hilfe des Sonnenkompasses feststellen, (b) die Entfernung nur aufgrund des während der Sammelflüge in unvergiftetem Zustand getroffenen Energieverbrauches messen. Im Zustand der Hyperaktivität (erhöhte Fluggeschwindigkeit) ist die Energiemenge bereits früher verbraucht, d. h. die Bienen suchen die Futterquelle in einer kürzeren Entfernung. Damit sind die Befunde von Heray und Mitarbeitern und Bisetzky erneut bestätigt. Gegen den möglichen Einwand, die Bienen seien nur deshalb nicht zum Dressurpunkt gelangt, weil sie vorher bereits auf die Duftplatten gestossen waren, sprechen mindestens drei Punkte :

1. Unter diesen Umständen hätte die Mehrzahl an dem vom Stock nächstentfernten Beobachtungspunkt in 420 Meter registriert werden müssen.

2. Die Lage der Duftplatten war bereits fester Bestandteil der Dressur (Abbildung $12: A$ ), d. h. die Sammlerinnen überflogen während ihrer Sammelflüge zur Futterquelle immer wieder die ausgelegten Duftplatten, ohne sie ebenfalls als Futterquelle betrachtet oder gar kennengelernt zu haben.

3. Vergleicht man die für die einzelnen Flüge zu den Beobachtungspunkten benötigten Zeiten, so fällt auf, daß die bei 550 Meter registrierte Biene bereits gelandet war, bevor die erste Biene an der Platte bei 480 Meter sich niederließ. 
Kehren wir jedoch zurück zu dem Thema : Tanztempo. Aus den Abbildungen 7 und 9 ist ersichtlich, daß vergiftete Sammlerinnen für einen Futterplatz in ca. 600 Meter mit einem Tanztempo werben, das dem unvergifteter Bienen für ca. 420 Meter gleichkommt. Dreiviertel der vergifteten Dressurbienen haben allerdings die Duftplatte bei 480 Meter angeflogen. Davon können zwei Folgerungen getroffen werden :

a. Die Einschätzung einer Entfernung durch vergiftete Bienen stimmt nicht mit dem Tanztempo überein, welches für die eingeschätzte Entfernung oder gar die tatsächliche Entfernung zuträfe. Die von uns untersuchten Bienen der in den Abbildungen 3 bis 10 wiedergegebenen Experimente fanden nach ihrer Vergiftung den Futterplatz sicher aufgrund der ihnen wohlbekannten Landmarken wieder.

$b$. Es ist denkbar, daß Tänzerinnen nach der Vergiftung die Entfernung für eine Futterquelle « subjektiv » in einem langsameren Tanztempo angeben. Diesem Tempo wirkt jedoch die erhöhte Laufgeschwindigkeit derart entgegen, daß es in summa zu einem schnelleren Tanztempo als dem in unvergiftetem Zustand kommt.

In umfangreichen Untersuchungen (HeraN 1956, Еsch 1956a, 1956b, 1961a, 1961b, 1964, Steche 1957, WenNer 1962) und Berechnungen (v. Frisch u. JANDER 1957) wurden die im Schwänzeltanz enthaltenen “ Einzelelemente » auf ihren Signalwert für die Entfernungsweisung hin untersucht und gegeneinander gewertet. Danach scheint die Schwänzelzeit das maßgebende Signal für die Entfernung zu sein. Der Signalwert der gesamten Umlaufzeit steht allerdings nur wenig hintenan.

Eine gegenseitige Aufrechnung von Tanztempo, Laufgeschwindigkeit und Energieverbrauch könnte unsere Überlegungen erhärten. Dazu sind jedoch zahlreiche derartige Versetzungsversuche bei unterschiedlicher Dressurentfernung notwendig.

\section{VII. - DIE VERGIFTUNGSERSCHEINUNG UND DIE DAUER IHRER WIRKUNG}

Am Schluß dieser Untersuchungen sei noch die Frage gestellt, ob die Vergiftung - es handelt sich ja lediglich um Bruchteile eines millionstel Grammes - den Vorgang der Entfernungsweisung permanent schädigend beeinflußt, oder ob die Bienen nach einer bestimmten Zeitperiode wieder zu ihrem ursprünglichen Tanztempo zurückkehren. Veränderungen in Bezug auf die Richtungsweisung sind spätestens nach 23 Stunden verschwunden (SCHRICKER u. Stephen 1970).

Setzt man einen Versuch zur Tanztempomessung über mehrere Stunden fort, so wird offensichtlich (Abb. 13), daß das Tanztempo der vergifteten 


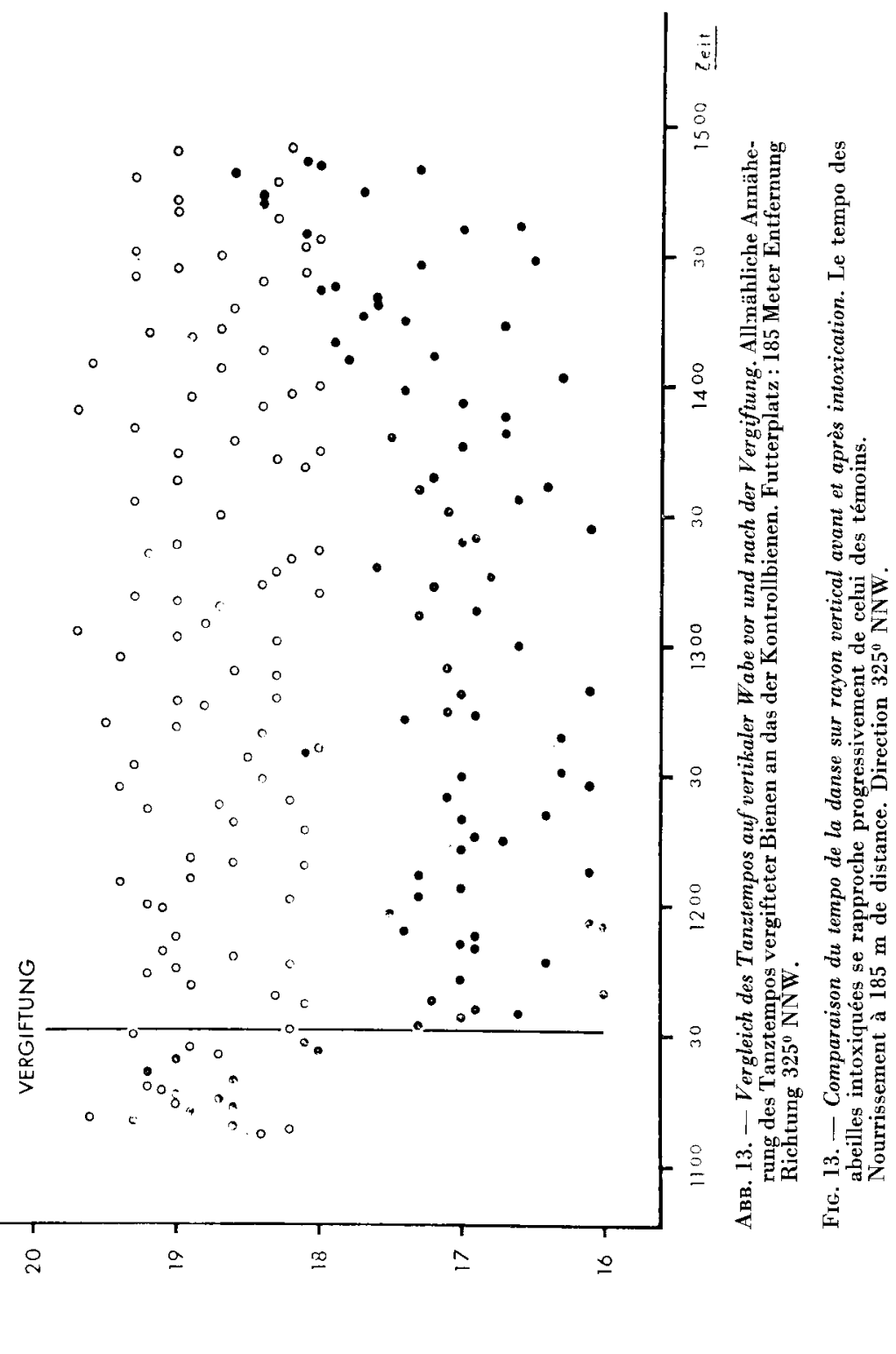



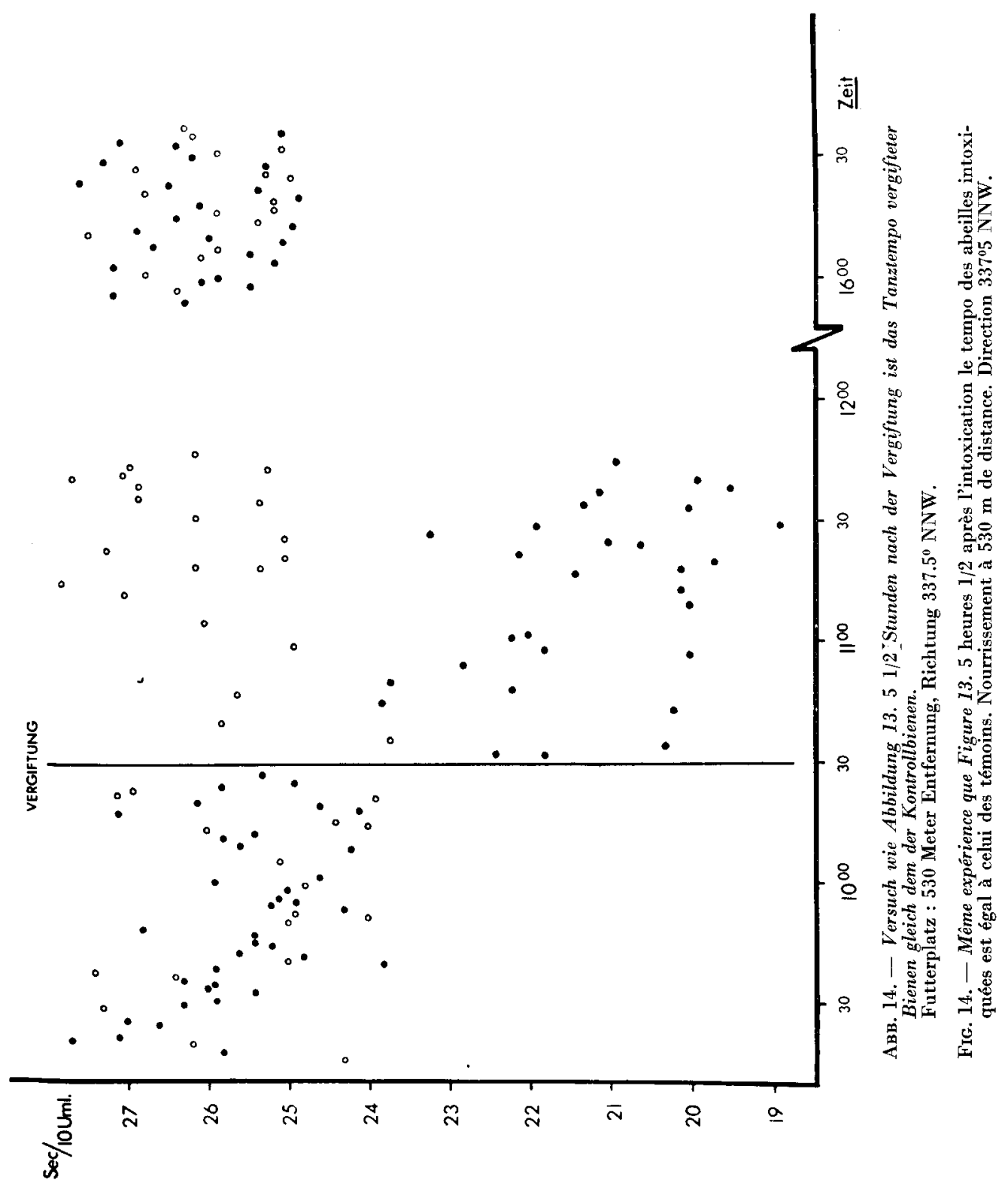
Sammlerinnen nach 2.5 bis 3 Stunden wieder langsamer wird (hier bei einem Futterplatz in 185 Meter Entfernung), und nach 5.5 Stunden (Abb. 14, für eine Futterquelle in 530 Meter Entfernung) ist es nicht mehr von dem unvergifteter Kontrollbienen verschieden.

Inwieweit es sich bei den letzten Versuchen um die Folge des Giftabbaues im Körper oder um Kompensationsvorgänge handelt, kann nicht entschieden werden. In diesem Punkt ist bisher zu wenig bekannt. Auch in Hinblick auf die Erklärung des Wirkungsmechanismus von Parathion bei Insekten ist man noch sehr auf Vergleiche von Einzelergebnissen angewiesen, obwohl hiervon eine ganze Fülle - allerdings vornehmlich von Orthopteren, Blattoiden und Dipteren - vorliegt (Zusammenfassung : Metcalf 1955, Calhoun 1963, Boistel 1968).

Parathion (Diäthyl-p-nitrophenylthiophosphat) gehört zu der Giftklasse der organischen Phosphorverbindungen, deren hauptsächliche Giftwirkung - soweit bekannt - darin besteht, daß die bei der Erregungsleitung am Nerven notwendige Cholinesterase oder funktionsähnliche Substanzen (für Apis : Augustinsson u. Grahn 1954) gehemmt werden (Narahashi 1971). An den motorischen Endplatten beispielsweise findet keine Unterbrechung der Erregungsleitung aufgrund der nicht mehr möglichen Spaltung des Azetylcholins statt. Die Folge sind andauernde Muskelkontraktionen (Muskelzittern), die bis zum Tetanus führen können. Metcalf u. March (1949) zeigten eine direkte Beziehung zwischen der Vergiftungsrate von Bienen mit Parathion der Hemmung von ACh - Esterase im Gehirn auf : Eine topikal verabfolgte Giftgabe von 0.1 Gamma wirkte letal. Die Hemmung der ACh - Esterase im Gehirn solcher Bienen betrug 90 bis $94 \%$. Hyperaktivität der Tiere wurde bei einer ACh - Esterase-Hemmung von $50 \%$ festgestellt. Eine Umrechnung auf die entsprechende Giftmenge führt zu 0.05 Gamma. Dieser Wert (nach topikaler Vergiftung) kommt unserer Bestimmung der subletalen Giftmenge von ( unter 0.03 Gamma ) sehr nahe.

Es erscheint im Falle der bisherigen Untersuchungen sehr schwer, die verschiedenen Angriffsorte des Giftes im Organismus « Biene » zu lokalisieren. Sie liegen offensichtlich nicht nur an den Verbindungsstellen Sinnesorgan Nervensystem bzw. Nervensystem - Erfolgsorgan, sondern ebenso an zentralen Synapsen, worauf unsere Untersuchungen zur Richtungsweisung (Schricker u. Stephen 1970, Stephen u. Schricker 1970) und zum Zeitgedächtnis der Bienen (SCHRICKER, in Vorbereitung) hinweisen.

Eingegangen im Februar 1974.

Reçu pour publication en février 1974.

\section{DANKSAGUNG}

Ich danke herzlich Frau A. Großkopf-Friauf für ihre Mitarbeit, Kollegen an der Oregon State University (Corvallis, Oregon U.S.A.) für ihre Beteiligung an den Versetzungsversuchen und Frau C. St. Friedemann für die Anfertigung von Zeichnungen. 


\section{RÉSUMÉ}

Les présentes recherches mettent en évidence l'action de très faibles doses de parathion (E 605) sur différents processus de comportement et notamment sur les paramètres de la danse frétillante des abeilles qui sont en rapport avec l'indication de la distance. Des recherehes antérieures (Schricker et Stephen, 1970; Stephen et Schricker, 1970) avaient déjà montré des modifications de l'indication de la direction consécutives à une intoxication des danseuses par des doses sublétales de parathion.

La dose de poison sublétale administrée par voie orale dans les présents essais est inférieure à 0,03 gamma (Fig. 2). Les butineuses dressées sur une source de nourriture artificielle indiquent, après leur intoxication, une distance entre cette source et la ruche qui est trop courte, ce qui s'exprime par un tempo plus élevé.

Dans ce cas il n'y a pas de différence, que la danse soit effectuée sur un rayon vertical (Fig. 3-7) ou horizontal (Fig. 8-9). Les novices ne se rendent pas compte de la modification des danses des butineuses intoxiquées et, en conséquence, elles sont guidées de façon erronée (Fig. 10).

Des butineuses, bien entraînées à visiter une source de nourriture artificielle sont intoxiquées sur place et, immédiatement après, transportées sur un terrain étranger. Sur ce nouveau terrain elles cherchent de même la source de nourriture à une distance plus courte (Fig. 12).

Dans des expériences de marche phototaxique vers une source lumineuse artificielle, la vitesse de marche des abeilles intoxiquées est supérieure de $10 \%$ environ à celle qu'on trouve à l'état non-intoxiqué (Fig. 11). L'action d'une dose sublétale de parathion sur le temps de la danse s'atténue 2 heures $1 / 2$ environ après l'intoxication et, après 5 heures $1 / 2$, elle ne peut plus être mise en évidence (Fig. 13-14).

Le mécanisme possible de l'action du parathion dans ces essais est discuté et comparé à celui de l'inactivation de l'acétyl-choline-estérase dans le cerveau de l'abeille étudié dans d'autres expériences. Enfin, le lien entre l'indication de la distance et le temps de la danse est discuté et interprété sur la base des résultats d'expériences antérieures.

\section{LITERATURVERZEICHNIS}

Augustinsson, K. B. u. M. Grahn : The Occurence of Cholinesters in the Honey Bee. Acta physiol. scand. 32, 174-190 (1954)

Bisetzky, R. : Die Tänze der Bienen nach einem Fußweg zum Futterplatz. Z. vergl. Physiol. 40, 264-288 (1957)

Bосн, R. : Rassenmäßige Unterschiede bei den Tänzen der Honigbiene (Apis mellifica L.). Z. vergl. Physiol. 40, 289-320 (1957)

Boistel, J. : The Synaptic Transmission and Related Phenomena in Insects. Advances in Ins. Physiol. 5, 1-64. (1968)

BräUninger. H. D. : Über den Ein fluß metereologischer Faktoren auf die Entfernungsweisung im Tanz der Bienen. Z. vergl. Physiol. 48, 1-130 (1964)

Calmoun, E. H. : The Physiological Significance of Acetylcholine in Insects and Observations upon other Pharmacologically Active Substances. Advances in Ins. Physiol. 1, 1-46 (1963)

Garson, R. : The Silent Spring. Boston (1962).

Esch, H. : Analyse der Schwänzelphase im Tanz der Bienen. Naturwissenschaften 43, 207 (1956a).

- Die Elemente der Entfernungsmitteilung im Tanz der Bienen. Experientia 12, 439-441 (1956b). 
- Ein neuer Bewegungstyp im Schwänzeltanz der Bienen. Naturwissenschaften 48, 140-141 (1961a).

- Über die Schallerzeugung beim Werbetanz der Honigbiene. Z. vergl. Physiol. 45, 1-11 (1961b).

- Beiträge zum Problem der Entfernungsweisung in den Schwänzeltänzen der Honigbienen. Z. vergl. Physiol. 48, 534-546 (1964).

- u. J. A. Bastian : How do Newly Recruited Honey Bees Approach a Food Site? Z. vergl. Physiol. 68, 175-181 (1970).

Floney, E. : Experimentelle Erzeugung einer « Neurose » bei der Honigbiene. Naturwissens. chaften 41, 171 (1954).

Frisch, K. v. : Tanzsprache und Orientierung der Bienen. Berlin-Heidelberg-New York (1965).

- u. M. Lindauer : Über die Fluggeschwindigkeit der Bienen und ihre Richtungsweisung bei Seitenwind. Naturwissenschaften 42, 377-385 (1955).

— u. R. JANDER : Über den Schwänzeltanz der Bienen. Z. vergl. Physiol. 40, 239-263 (1957).

Heran, H. : Versuche über die Windkompensation der Bienen. Naturwissenschaften 42, 132-133 (1955).

- Ein Beitrag zur Frage nach der Wahrnehmungsgrundlage der Entfernungsweisung der Bienen. Z. vergl. Physiol. 38, 168-218 (1956).

- Wie beeinflußt eine zusätzliche Last die Fluggeschwindigkeit der Honigbiene? Zool. Anz. 26, Suppl. Bd. 346-354 (1963).

- Der Insekten flug und seine nervöse Steuerung. Naturwiss. Rdsch. 22, 1-8 (1969).

- u. L. WaNke : Beobachtungen über die Entfernungsmeldung der Sammelbienen. Z. vergl. Physiol. 34, 383-393 (1952).

- u. E. Schreindeer : Das Tanztempo der Honigbiene und ihre Flugerfahrung. Z. vergl. Physiol. 54, 165-191 (1967).

Lindauer, M. : Über die Einwirkung von Duft- und Geschmackstoffen sowie anderer Faktoren auf die Tänze der Bienen. Z. vergl. Physiol, 31, 348-412 (1948).

- Schwarmbienen auf Wohnungssuche. Z. vergl. Physiol. 37, 263-324 (1955).

- The Functional Significance of the Honeybee Waggle Dance. The Amer. Naturalist 105, $89-96$ (1971).

- u. J. O. Nedel : Ein Schweresinnesorgan der Honigbiene. Z. vergl. Physiol. 42, 334-364 (1959).

Metcalf, R. L. : Organic Insecticides - Their Chemistry and Mode of Action. New York (1955).

- u. R. B. March : Studies of the Mode of Action of Parathion and Its Derivates and Their Toxicity to Insects. J. Econ. Ent. 42, 721-728 (1949).

Narahashi, T. : Effects of Insecticides on Excitable Tissues. Advances in Ins. Physiol. 8, $1-93$ (1971).

Newson, L. D. : Consequences of Insecticide Use on Nontarget Organisms. Ann. Rev. Ent. $12,257-286$ (1967).

P̈̈тAU, K. : Zur statistischen Beurteilung von Meßreihen (eine neue t-Tafel). Biol. Zbl. 63, 152-168 (1943).

Perkow, W. : Die Insektizide. Heidelberg (1956).

Schick, W. : Über die Wirkung von Giftstoffen auf die Tänze der Bienen. Z. vergl. Physiol. 35, 105-128 (1953).

Schifferer, G. : Zitiert bei K. v. Frisch (1965).

Scholze, E., H. Pichler u. H. Heran : Zur Entfernungsschätzung der Bienen nach dem Kraftaufwand. Naturwissenschaften 51, 69-70 (1964).

Schricker, B. : Der Einfluß subletaler Dosen von Parathion auf das Zeitgedächtnis der Honigbiene. Apidologie 5 (i. Druck).

- u. W. P. Stephen : The Effect of Sublethal Doses of Parathion on Honeybee Behaviour. I. Oral Administration and the Communication Dance. J. Apic. Res. 9, 141-153 (1970).

Steche, W. : Beiträge zur Analyse der Bienentänze. Ins. Sociaux 4, 305-318 (1957). 
Stephen, W. P. u. B. Schricker : The Effect of Sublethal Doses of Parathion on Honeybee Behaviour. II. Site of Parathion Activity, and Signal Integration. J. Apic. Res. 9, 155-164 (1970).

Scrweıger, E. M. : U̇ber individuelle Unterschiede in der Entfernungs- und Richtungsangabe bei den Tänzen der Bienen. Z. vergl. Physiol. 41, 272-299 (1958).

Wenner, A. M. : Sound Production During the Waggle Dance of the Honey Bee. Anim. Behav. 10, 79-95 (1962).

- The Bee Language Controversy — an Experience in Science. Denver (1971)

Werner, G. Tänze und Zeitempfinden der Honigbiene in Abhängigkeit vom Stoffwechsel. Z. vergl. Physiol. 36, 464-487 (1954).

WITT, P. N. u. C. F. REED : Spider-web building. Measurement of Web Geometry Identifies Components in a Complex Invertebrate Behavior Pattern. Science 149, $1190-1197$ (1965).

- Ch. F. Reed u. D. B. Peakall : A Spider's Web. Berlin-Heidelberg-New York (1968).

Young, C. L. u. W. P. StePhen : The Acoustical Behavior of Acheta domesticus L. (Orthoptera : Gryllidae) Following Sublethal Doses of Parathion, Dieldrin and Sevin. Oecologia 4, 143-162 (1970). 